\title{
MARSTON MORSE AND HIS MATHEMATICAL WORKS
}

\author{
BY RAOUL BOTT ${ }^{1}$
}

1. Introduction. Marston Morse was born in 1892, so that he was 33 years old when in 1925 his paper Relations between the critical points of a real-valued function of $n$ independent variables appeared in the Transactions of the American Mathematical Society. Thus Morse grew to maturity just at the time when the subject of Analysis Situs was being shaped by such masters ${ }^{2}$ as Poincaré, Veblen, L. E. J. Brouwer, G. D. Birkhoff, Lefschetz and Alexander, and it was Morse's genius and destiny to discover one of the most beautiful and far-reaching relations between this fledgling and Analysis; a relation which is now known as Morse Theory.

In retrospect all great ideas take on a certain simplicity and inevitability, partly because they shape the whole subsequent development of the subject. And so to us, today, Morse Theory seems natural and inevitable. However one only has to glance at these early papers to see what a tour de force it was in the 1920's to go from the mini-max principle of Birkhoff to the Morse inequalities, let alone extend these inequalities to function spaces, so that by the early 30's Morse could establish the theorem that for any Riemann structure on the $n$-sphere, there must be an infinite number of geodesics joining any two points.

This whole flight of ideas was of course acclaimed by the mathematical world. It brought him to the Institute for Advanced Study in 1935, when, at 43, he also delivered the Colloquium Lectures of the Mathematical Society and wrote his monumental book on the Calculus of Variations in the Large; it eventually earned him practically every honor of the mathematical community, over twenty honorary degrees, the National Science Medal, the Legion of Honor of France, .....

Nevertheless, when I first met Marston in 1949 he was in a sense a solitary figure, battling the algebraic topology, into which his beloved Analysis Situs had grown. For Marston always saw topology from the side of Analysis, Mechanics, and Differential geometry. The unsolved problems he proposed had to do with dynamics-the three body problem, the billiard ball problem, and so on. The development of the algebraic tools of topology, or the project of bringing order into the vast number of homology theories which had sprung up in the thirties-and which was eventually accomplished by the Eilenberg-Steenrod axioms-these had little interest for him. "The battle between algebra and geometry has been waged from antiquity to the present" he wrote in his address Mathematics and the Arts at Kenyon College in 1949, and

\footnotetext{
Received by the editors April 15, 1980.

'This work was supported in part through funds provided by the National Science Foundation under the grant 33-966-7566-2.

${ }^{2}$ Poincare was born in 1854 , the others all in the 1880 's. 
again, a few lines later: "Forever the foundation and never the Cathedral."

Poincaré's interest in Analysis Situs had two distinct sources: Dynamics and Algebraic Geometry. And these two mainsprings of Topology were magnificently represented by G. D. Birkhoff and S. Lefschetz respectively, in the early part of this century. Through his teacher, G. D. Birkhoff, Morse had inherited the dynamical tradition-in no uncertain terms- and it was in this framework that he understood Analysis Situs.

There was thus a natural ambivalence to him to the sibling algebraic branch which had evolved so strongly around Lefschetz in Princeton. I distinctly recall an afternoon in 1949 when he reminisced about his appointment at the I. A. S. and spoke wistfully about how differently things would look if he had gone to the University and Lefschetz had taken on the post at the Institute. His wrath, I should hasten to add, was never personal-and certainly not directed at Lefschetz's achievements in algebraic geometry. Rather, he resented the omnipresence of algebra in the topological scene at that time. And certainly it was true that in 1949 the Geometric tradition of topology was not nearly as well represented as it is, say, today. And it is also true that at decisive moments it was precisely the Morse Theory-in the highly geometric setting of Smale-which overcame the greatest obstacles. On the other hand the late forties and early fifties were exciting years for homotopy theory; for with the advent of the algebraic tools of the Steenrod Algebra and Serre's application of the Leray Spectral Sequence homotopy theory had become tamed, and it is not surprising that this development temporarily eclipsed all others.

The next sections will be devoted to a more detailed account of the Morse theory and other aspects of Morse's work, but before going on, I find the urge to reminisce-once indulged in-too strong to be denied. It was my good fortune to come to the Institute for Advanced Study in Princeton in 1949, largely through the good offices of my teacher R. Duffin at Carnegie Institute of Technology and of $\mathrm{H}$. Weyl, who had befriended me there in Pittsburgh while visiting Carnegie on a lecture tour. The general plan of my appointment as I understood it, was that I was to write a book on network theory at the Institute.

I suppose that a young prospective knight approaching King Arthur's table for the first time must have felt as I did when I first walked into Fuld Hall and took possession of my small office on the third floor. The professors of Mathematics at that time were Oswald Veblen, Hermann Weyl, John Von Neumann, Carl Ludwig Siegel, Marston Morse and James W. Alexander. On the ground floor you passed Einstein's office and you were welcomed upon arrival by J. Robert Oppenheimer. The permanent members were Kurt Gödel, Deane Montgomery, and Atlè Selberg. The officer in charge of the temporary members that year was Marston Morse and it was in that capacity that I first met him. His office was also on the third floor and I had seen him bounding up the stairs several times before my official "reporting to work", so to speak, occurred.

The overwhelming impression which remains with me to this day is the vast energy which Marston somehow radiated. I compute now-with amazement-that he must have been fifty-seven at that time, and recall with some 
humiliation that a few weeks later at a party at our house, he easily beat me in a hundred yard dash to which he had-characteristically-challenged me. But to return to my "reporting to work". I approached this interview with some nervousness, because in the few weeks which I had spent at Princeton before it took place, the whole mysterious world of pure mathematics had burst upon me and all I wanted to do was explore it. In no way did I want to write the book.

Well, after five minutes with Marston all my uneasiness had vanished. First of all I found that I really did not have to say very much! I think it is a fair statement that in all conversations with Marston, one only had to do twenty percent of the talking. His energy was such that it just naturally took over. He immediately dismissed my fears of having to write a book. It was a matter of course to him that at the Institute a young man should only do what he wanted to do; that this was the place where a young man should find himself, and the last place in the world for performing a chore. And once this technical part of our interview was over he immediately, again characteristically I think, started to speak about the subject that absorbed his interest at the time. Actually, in 1949 this subject had nothing to do with critical point theory. Rather, he was deeply involved in his work with Transue, on functions of bounded variations. In any case, I remember leaving this interview with a light heart, newly liberated and buoyed by the energy and optimism I had just encountered. I was also elated by the directness of Marston's manner. There was not the slightest condescension in it. Although he dominated-I expect-all encounters, he treated everyone as an equal, with complete honesty, and in personal matters he showed great kindness and generosity.

Mathematically, Marston and I did not communicate too well, and I don't think we could have collaborated. I also recall really only one private lecture on critical point theory from him. His primary interests were elsewhere at the time. But on the personal side we got on right from the start, even though we of ten disagreed.

Marston loved music and played the piano beautifully and effortlessly. He was devoted to Bach and very knowledgeable about all aspects of music, and so music was our first and quite natural bond. But beyond that and quite apart from certain affinities of taste, I think I immediately sensed and revered his spiritual nature. Marston was a deeply religious man, yet I never heard him "preach". One was conscious of this aspect of his life only indirectly and quite marvelously. His daughter-in-law, Terry Morse, put it better than I every could.

"His personality had a light and a force which was very spiritual and mysterious. I think it was because he welcomed the ultimate mystery of life, embraced it, and took great joy in it, that we always came away from being with him feeling a heightened sense of awareness of the beauty and the possibilities in life," she wrote to Louise Morse after his death.

And then, there was his wonderful wife Louise, to whom we-indeed all of us new green Ph.D.'s-were immediately drawn, and who was such a natural complement to Marston. The Morses took their stewardship of the mathematical community very seriously and it was to them we turned in times of 
need. Of course Marston's advice could be disheartening. I remember calling on him for suggestions concerning a summer job in 1950. After some reflection he recommended mowing lawns or baby sitting! When it came time to move on in 1951, he would recommend the mathematical wasteland. "Get away from Princeton and the mathematical centers. Have your own thoughts in peace and quiet," he would say. "Look what Lefschetz did in Kansas."

But it is time to hear different memories of the past than mine. Maurice Heins, who knew Marston as a student and collaborator and friend, writes as follows:

"My first encounter with Marston was as a sophomore in what was then Mathematics 5 at Harvard. It was his last year at Harvard. Characteristic of his lectures were lucidity, simplicity and eloquence. One felt that one was in the presence of a master. He shared with Heinz Hopf a strongly tactile presentation. It was as if they were sculptors in their ateliers. In this connection, I recall that Marston thought that his experience with carpentry contributed to his geometric sense greatly. In 1934 the curriculum of a course like Math 5 was extremely rigid. Marston conveyed very beautifully and intuitively without belaboring the $\varepsilon$ 's the import and the essential ideas of the proofs of such theorems as the implicit function theorem, the Sturm separation theorem (curriculum not withstanding), the Euler-Lagrange condition. One has to keep in mind the quasitotal absence of mathematical sophistication of the part of his hearers."

"What was very impressive about Marston in the 20's and 30's was that he was devoid of the bigotry that paraded in the guise of gentility during that period. He valued one's worth and integrity, not the accidentals of birth. He had enormous drive and the physical capacity for many hours of work. Twenty hour work days were common."

"The intensity of his devotion to scholarship was coupled with uncompromising standards of rigor imposed both on himself and his collaborators. Working with him was a very intense experience."

"Our collaborative work was proposed by Marston who wanted to bring critical point theory to bear on the theory of harmonic functions and analytic functions, or, more generally, the pseudoharmonic functions and light interior transformations. The physical circumstances of the collaboration may appear surprising but exemplify some of the things said above about Marston's temperament. At the tme he was in Washington as a scientific consultant to the then War Department, Office Chief of Ordnance. I was a P-4 mathematician working with him and W. R. Transue. After the normal work day Marston and I worked on the joint papers from 7 to 11, Monday through Thursday. He wrote drafts of the work on the weekends back in Princeton ..."

I quote next from William Transue's reminiscences:

"In the fall of 1942 I went to the Institute as Marston's assistant. I was a fresh Ph.D., with dissertation on subharmonic functions, but with no set direction except for the broad field of analysis. At that time Marston had the idea of applying topological methods to obtain information on the 3-body problem, and he set me to work reading F. R. Moulton's Celestial mechanics and a paper of G. D. Birkhoff in this area. However, Marston was at this time 
a consultant to the Office of the Chief of Ordnance and spent more and more time in Washington. In the spring of 1943 I moved to Washington and all of my attention and most of his was devoted to military problems. These were "applied" mathematics of the dirtiest sort, applied to whatever difficulties the Ordnance Department was encountering, but came to center principally on the area known as terminal ballistics-the study of the destructive effects of bombs and shells. The mathematics involved were usually of the most elementary sort, generally numerical integrations, and we were delighted when we could bring in something as sophisticated as the icosahedron. This was before the day of the computer, and our computations were done by a small staff using keyboard machines to add and subtract, multiply and divide. Programming computations for such a staff was roughly equivalent to programming a present-day computer. Marston's capacity for sheer hard, and usually dull, work on these problems was unbelievable. After the offices were closed in the evening we would go on for a couple of hours. I remember particularly one set of data on land mine explosions which seemed to make no good sense. The rest of us were ready to give up, but Marston returned to the attack again and again, determined to beat some kind of order into the data, and we did finally put out some kind of analysis."

"Several years later we undertook the study of integral representation of bilinear functionals and allied topics which continued for some years. I spent one full year at the Institute and many summers with him at various places-Princeton, Cape Cod, Maine. Working with Marston (for me at least) meant being completely taken over, spending almost all waking hours with him, talking mathematics all day, including during many meals taken with the Morse household, and continuing late into the evening. He was a real bear for work. In Princeton we usually worked in his bedroom (Richard Arens once remarked that he couldn't concentrate with someone else's pants hanging in the closet!), and in Maine sometimes in the car. Louise was always extremely good about finding him a quiet place to work, but with the number of children about, this was not always easy. For his part, he was a very considerate husband and father, and the Morse household, which I got to know pretty intimately, was a very happy and harmonious one."

"The stamp of his Maine upbringing was pretty heavy on him, and he retained not only the industry, but the frugality which characterizes the Maine citizen. Although he gave his money generously, he spent it carefully. He was equally generous with his time. I recall one evening in Princeton, when Louise and the children were out of town and we were doing our own cooking. In the midst of warming our soup, the telephone rang and a reporter from Time magazine wanted Marston to explain the theory of relativity. Well, he did! He could never refuse to teach anyone."

"He was a great Francophile and liked most to think of himself as a mathematical descendant of Poincaré. His conversion to Catholicism brought him also particularly close to Italy. Of course, his position made him widely known in the international community of mathematics, but his feelings for France and Italy were special ...."

Finally I quote from a letter by $S$. Cairns, a life long friend and collabora- 


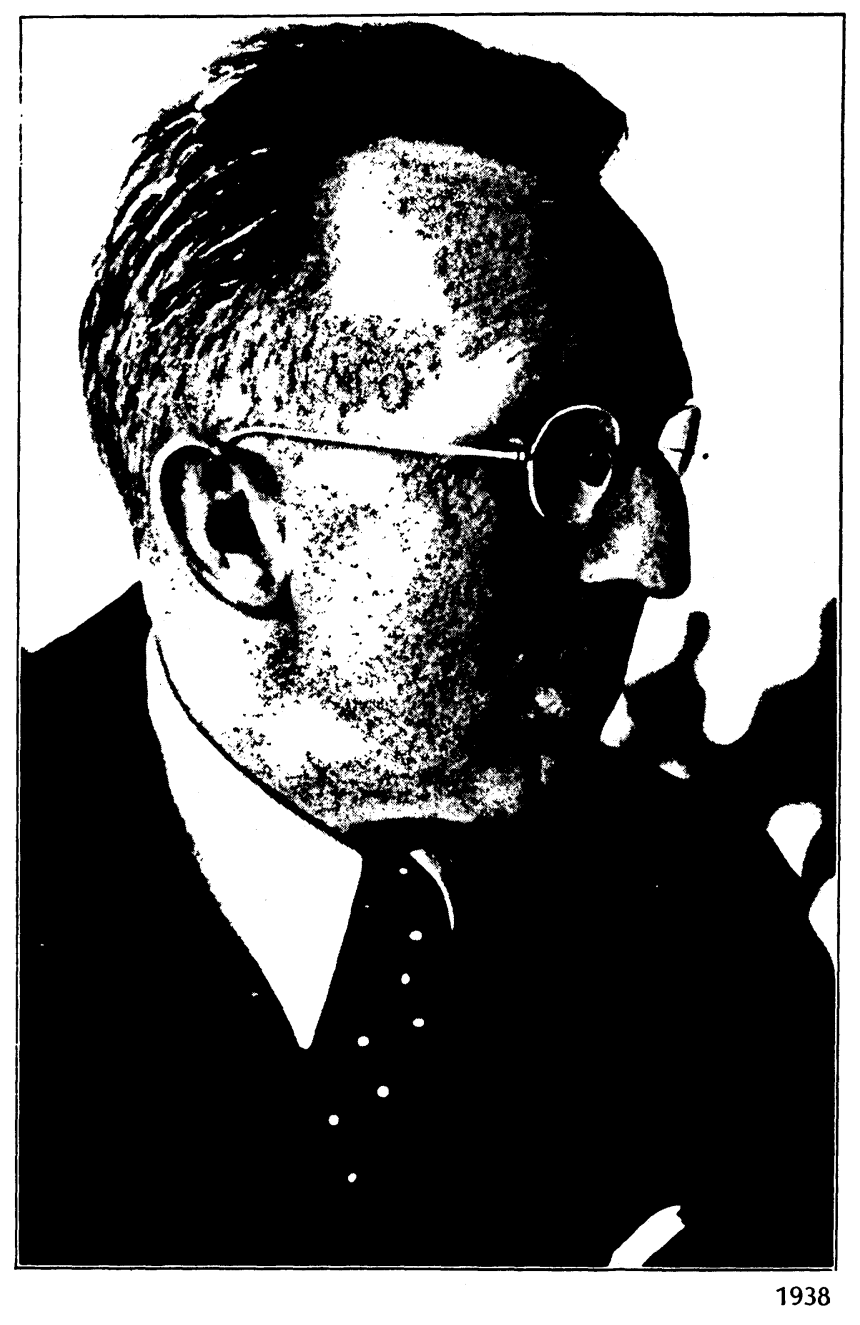

Maretor Moure.

$1892-1977$ 
tor, in which he gives a brief chronological account of Morse's career, as well as some personal remembrances.

"In the fall of 1926, after the completion of undergraduate work at Harvard, I made the acquaintance of Marston Morse. He had just joined the faculty of Harvard University. During the ensuing academic year, he conducted a lively seminar in topology. This subject, then in a relatively primitive stage, was essential to the research in global analysis on which he had embarked. In the following year, Morse suggested to me, as subject for a doctoral dissertation, the triangulation of the differentiable manifold, with the enticing comment that I could start on it at once because there was no literature to read. Thus commenced more than fifty years of professional association and personal friendship, culminating in collaboration during the last twelve years of Morse's life."

"As teacher and research worker for nine years at Harvard, Morse was a stimulating source of enlightenment and inspiration. He saw mathematics as a challenging thing of beauty and imparted his enthusiasm to others."

"Morse was born in 1892 in Waterville, Maine, where he had his early education and where he completed his undergraduate work in 1914 at Colby College. Three years later he received the degree of Ph.D. from Harvard, having meanwhile published his first research paper in 1916."

"World War I interrupted his career. He served with distinction in the American Expeditionary Force and was awarded the Croix de Guerre with Silver Star for bravery under fire."

"Resuming the academic life, Morse taught at Cornell, 1920-1925, and at Brown, 1925-1926, before his appointment to Harvard University."

"In 1935, Morse accepted a professorship at the Institute for Advanced Study, which had just been established in Princeton to provide an ideal environment for the most active and distinguished scholars in a broad spectrum of disciplines. Morse retired in 1962 as professor emeritus, but, for the rest of his eighty-five years, continued his research with extraordinary vigor and creativity."

"In World War II, Morse was a consultant in the Office of the Chief of Ordnance. His invaluable work on military applications of mathematics was recognized by a Meritorious Service Award, conferred in 1944 by President Roosevelt."

"After the war, he was a prime mover in the creation of the National Science Foundation. President Truman appointed him to serve on its first board from 1950 to 1954 ."

"In 1952, Morse was a representative of the Vatican at the Atoms for Peace Conference of the United Nations."

"He was president of the American Mathematical Society, 1940-1942; a vice president of the International Mathematical Union, starting in 1958; chairman of the Division of Mathematics of the National Research Council, 1951-1952. The list of such offices could be continued. In his community he served on the boards of two private schools and of the Princeton Chapter of Recording for the Blind."

"Many honors were bestowed on Morse, among them honorary degrees from twenty institutions in the U.S.A., Austria, France and Italy. These 
included the University of Paris (1946), Pisa (1948), Vienna (1952), Harvard (1965) and Modena (1975). In 1952, he became a Chevalier of the French Legion of Honor. He was elected in 1932 to the National Academy of Sciences and in 1956, as an associate member, to the French Academy of Sciences. His affinity for France made the honors from that country particularly gratifying. He also cherished his election as a corresponding member of the Italian National Academy Lincei. A National Medal of Science was awarded to him in 1964 and presented by President Johnson at the White House in 1965. Again the list could be prolonged."

"A year or two after his retirement, in a conversation, Morse outlined to me the problems he hoped to solve, if only he could live twenty years more and keep on doing research. It has been gratifying to see a substantial part of his hope fulfilled and to collaborate with him in its implementation."

"Essential to the remarkable prolongation of his long and brilliant career was the devoted care and sympathetic understanding of Mrs. Morse. She was the mistress of their home, the mother of their five children and a charming hostess to countless colleagues and friends. Also, with tender skill, she helped conserve her husband's health in his advancing years."

"Besides Mrs. Morse and their children Julia, William, Elizabeth, Peter and Louise there survives one of the two offspring of an earlier marriage, Dr. Dryden Morse. Meroë, his eldest daughter, died in 1969."

"Morse's cultural interests extended far beyond the boundaries of his profession. Music, in particular, was a lifelong avocation. He played the piano with consummate skill and sensitivity. His repertory of classical music was large and was increasing up to the end ...."

"Science, philosophy, religion and the arts were objects of Morse's inquiring mind. He recognized the fundamental unity of creativity in all these areas. A stimulating treatment of this subject is to be found in his paper, Mathematics and the Arts, Bulletin of the Atomic Scientists 9 (1959), 55-59, based on his lecture at Kenyon College in 1949, during a conference honoring Robert Frost ...."

A moment I will always cherish occurred upon Marston's return from his lecture at Kenyon. He met me as he came bounding up the stairs to our third floor, and immediately took me into his office to tell me what a marvelous speech he had given: "The speaker before me had a terrible voice," he remarked, "And really didn't have much to say, so when I finished, I brought the house down."

And of course he is quite right; his essay is a masterpiece, and had indeed brought the house down.-There was no modesty in Marston; he told it as he saw it.

His life was gentle, and the elements

So mixed in him that nature might stand up

And say to all the world, "This was a man".

2. The works of Marston Morse. It would be impossible to comment in detail on the bulk of Morse's work. His bibliography has 180 entries, and includes seven books. Rather, I have selected eight topics which played a 
central role at various stages of his career and I will attempt to explain some aspects of each of these in detail.

Morse had many collaborators, and I have therefore included the names of his principal collaborators in the various subjects as well as a rough indication of the time when this work was done in the following list of the topics.

(3) Dynamics, geodesic flow (Hedlund, (1917-1940's))

(4) Morse theory (1921-1978)

(5) Minimal Surfaces (Tompkins, 1940's)

(6) Topological methods in a single complex variable (M. Heins, 1940's)

(7) Integral representations (Transue, 1940's, 1950's)

(8) Pseudoharmonic functions (Jenkins, 1950's)

(9) Differential topology (Hubsch, Cairns, 1960's-1970's)

Finally a word of thanks to the many colleagues who have helped in this enterprise. I am especially indebted to the collaborators of Morse already mentioned as well as: G. Mackey, D. Gromoll, J. Mather, D. Kazhdan, M. Brin, T. Goodwilly and N. Hingston.

3. Dynamics-geodesic flow. There are two outstanding results in Morse's work on this subject, and I will report on them under two headings-The Morse Trajectory, and Instability implies transitivity.

3.1. The Morse Trajectory. This discovery goes back to Morse's thesis of 1917 and was published under the title, Recurrent geodesic on a surface of negative curvature [3].

In 1944, Hedlund and Morse collaborated on a paper [60] where this same construction solves a problem in unending chess and in the theory of semigroups. This "Morse trajectory" also occurs in Novikov's disproof of the Frobenius-Burnside conjecture [N1] and was there attributed to a Russian mathematician writing in 1939. The trajectory in question occurs in the paper cited above dealing with the behavior of geodesics on surfaces of negative curvature imbedded in three-space. In this context this trajectory solves a problem posed by Birkhoff [B1] in 1912.

The surfaces which Morse considers are of the type indicated in Figure 1,

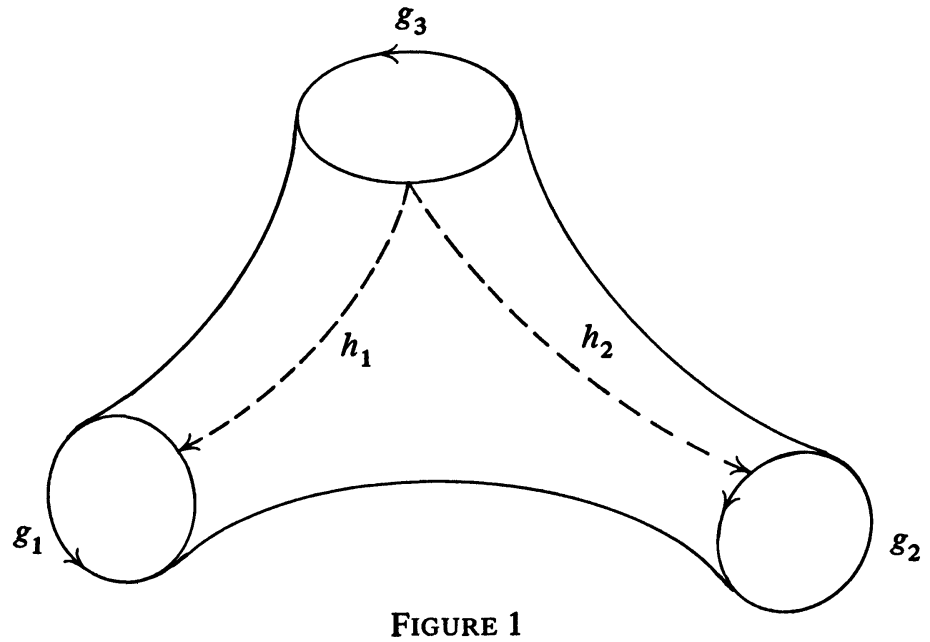


and in contemporary language are 2-manifolds with boundary, smoothly embedded in a compact region of $\mathbf{R}^{3}$ so as to inherit negative curvature except possibly at a finite number of points. The boundary circles are taken to be closed geodesics, and the genus of $S$ is assumed to be not less than 2

$$
\chi(S)>2 .
$$

Using the basic existence theorems of Hadamard $[\mathbf{H}]$, Morse gives a completely combinatorial description of those geodesics $g$ which lie entirely on $S$. Precisely, such a geodesic is then a map

$$
g: \mathbf{R} \rightarrow S
$$

satisfying the geodesic differential equation.

To every such "completely extended" geodesic Morse now assigns an object which nowadays we might call an infinite word in the generators of the fundamental group $\pi_{1}(S)$. Morse's strategy for doing this is to introduce geodesic segments $h_{1}, h_{2}$ as indicated above. Cut along these and $S$ becomes simply connected. Now any geodesic $g$ must cut these segments transversally at all points of intersection, and using this fact Morse assigns to $g$ a sequence of symbols

$$
\cdots C_{-2} C_{-1} C_{0} C_{1} C_{2} \cdots
$$

indexed by the integers, with each $C$ ranging over the set $g_{1}, g_{2} g_{1}^{-1}, g_{2}^{-1}$, with the understanding that a $g_{i}$ is never followed by a $g_{i}^{-1}$ and a $g_{i}^{-1}$ is never followed by a $g_{i}$. Morse calls such a sequence a Normal set and shows that modulo translation the Normal set gives a one-to-one representation of the geodesics entirely on $S$.

A slightly different, but quite equivalent description of the geodesic flow on $S$ had already been given by Morse in [2], but this normal set representation is the most useful for the purposes of this paper.

In modern language, this representation can be thought of this way: We first interpret the symbols $g_{1}$ and $g_{2}$ as generators of $\pi_{1}(S ; P)$, the fundamental group of $S$ based at $P$; thus think of $g_{1}$ as shorthand for the loop $h_{1}^{-1} \circ g_{1} \circ h_{1}$, etc. Then, once a starting point has been selected, $g$ gives rise to a nested sequence of geodesic segments with endpoints on $h_{1}$ and $h_{2}$, and thus to elements in $\pi_{1}\left(S^{1} ; P\right)$. These now have unique representations in the $g_{i}$ and so result in the unending normal sequence in question. I say in modern language, because in the papers under discussion one never encounters terms such as: Manifold, covering space, universal covering, group, or fundamental group, Euler Characteristic, etc. All these concepts were of course profoundly understood by the author and his contemporaries, but they do not seem to have crystallized into words at that time. Of course Morse had, in any case, an aversion to using technical terms which he did not coin himself.

But to return to the paper on recurrent geodesics, let me say one word of explanation about the inverse problem of constructing a geodesic $g$ from a given normal sequence. 
The proof of this fact depends on the following fundamental result of Hadamard: "Given a curve $c(t), 0<t<1$, on $S$ there is one and only one geodesic joining the endpoints of $c$ on $S$, which is homotopic to $c$ (with end points fixed). Furthermore this geodesic is of minimal length in its homotopy class". At this point the negative curvature of course enters vitally; this result is patently incorrect, say on the sphere.

The description of the geodesic flow on $S$ furnished by the Normal Sequences is the starting point of a discipline called "symbolic dynamics". It seems to have been independently discovered by various authors-but as far as I can see, always considerably later and usually in connection with surfaces of constant negative curvature. Marston Morse and Hedlund took up this subject in considerable detail much later in [39] and [43], where they developed delicate combinatorial criteria for symbolic trajectories; and of course by now this subject is well established in all aspects of the study of dynamical systems, see for instance [S4], [S5].

Some of the early consequences which Morse deduced from his representation are the following.

THEOREM. Every geodesic wholly on $S$ is the limit of closed geodesics.

Definition. A set $R$ of geodesics wholly on $S$ is called minimal if every element of $R$ has every other element of $R$ but no other geodesic as a limit geodesic.

Thus a closed geodesic is the simplest example of a minimal set. On the other hand Morse shows that

THEOREM. Every minimal set other than a closed geodesic has the power of the continuum.

And he then goes on to construct a nontrivial minimal set. By the way, he defines a geodesic to be recurrent if it is a member of such a minimal set. Thus in this terminology he constructs a recurrent geodesic which is not closed.

His method is of course to translate all these concepts into combinatorial form first, and then to construct a normal sequence with the desired combinatorial properties. The pertinent definitions are as follows.

A sequence

$$
\cdots R_{-2} R_{-1} R_{0} R_{1} R_{2} \cdots
$$

indexed by the integers is called recurrent if for every positive integer $r$ there is a positive integer $s$ such that every "segment" of length $r$

$$
R_{m} R_{m+1} \cdots R_{m+r}
$$

in (3.1) is contained in every segment of length $s$

$$
R_{n} R_{n+1} \cdots R_{n+s}
$$

of (3.1).

With this concept understood, Morse then constructs his ingenious Morse Trajectory on two symbols which he denotes 1 and 2-by the following 
inductive procedure (I quote verbatim): "Set

$$
\begin{aligned}
a_{0} & =1 \\
b_{0} & =2 \\
a_{1} & =a_{0} b_{0} \\
b_{1} & =b_{0} a_{0} \\
& \vdots \\
a_{n+1} & =a_{n} \cdot b_{n} \\
b_{n+2} & =b_{n} \cdot a_{n} .
\end{aligned}
$$

We introduce the set of symbols

$$
\cdots d_{-2} d_{-1} d_{0} d_{1} d_{2} \cdots
$$

of which $d_{0} d_{1} \cdots d_{2^{n}}$ are defined respectively as the $2^{n}$ integers of $a_{n}$; further if $m$ is any positive integer, $d_{-m}$ is defined as equal to $d_{m-1}$. The set (2.2) so defined will be proved to be recurrent without being periodic".

Explicitly

$$
\begin{array}{llll}
a_{0}=1, & a_{1}=12, & a_{2}=1221, & a_{3}=12212112, \\
b_{0}=2, & b_{1}=21, & b_{2}=2112, & b_{3}=21121221 .
\end{array}
$$

Note that if we replace 1 and 2 by $g_{1}$ and $g_{2}$ respectively, this Morse trajectory corresponds to a geodesic on the surface $S$ of Figure 1 and thus constitutes the first example of a set of "recurrent motions of discontinuous type" in the sense of Birkhoff [B2].

A final explicit result of the paper under discussion is the following.

THEOREM. On a surface $S$ of genus $\geqslant 2$ of the type we are considering, every geodesic wholly on $S$ is the limit of a recurrent but not periodic geodesic.

3.2. Instability and transitivity. On a Riemann manifold $M$, the "geodesic flow" defines an action of the real numbers $\mathbf{R}$ on the unit tangent bundle $T_{1} M$ of $M$ : The real number $r$ assigns to a tangent vector $X_{m}$ the unit tangent vector at the "final" end of a geodesic segment $s$ of length $r$ starting at $m$ and with initial direction $X_{m}$. The transitivity properties of this flow have been a subject of great interest throughout the last hundred years; in the period around 1930 especially, great advances were made with contributions from a veritable who's who of mathematicians of the period: Birkhoff, Hopf, Koopman, von Neumann, P. A. Smith. In 1934 Hedlund [H2] proved that this flow was metrically transitive (Ergodic) for any compact Riemann Surface $M$ of constant negative curvature. At the same time Morse in his paper [28] bearing the title of our heading, gave very much more general conditions on a surface $M$, for this flow to be topologically transitive.

Recall that in context, metrically transitive means that all $\boldsymbol{L}^{2}$-functions on $T_{1} M$ invariant under the flow are constant (up to a set of measure 0 ) while topological transitivity means that some orbit of the flow be dense in $T_{1} M$. By the Ergodic theorems of Birkhoff and von Neumann metric transitivity implies topological transitivity but not vice versa. 
Morse proves that a motion is topologically transitive provided it satisfies a uniform instability ${ }^{3}$ criterion, which he informally describes as follows: ". . . it may be roughly regarded as the hypothesis that the first conjugate point of any point $p$ on $M$ be beyond the point at $\infty \ldots$.. More precisely it is a hypothesis which ensures that neighboring geodesics diverge in a uniform manner. Technically it means the following: Suppose that $g: \mathbf{R} \rightarrow M$ is any geodesic parametrized by arc length and that $w(x)$ is any solution of the Jacobi equations along $g$

$$
d^{2} w / d x^{2}+k(x) w(x)=0,
$$

which satisfies the condition

$$
\left.(d w / d x)^{2}\right|_{x=0}+w(0)^{2}=1 .
$$

The geodesics on $M$ are then uniformly unstable if there are no pairs of conjugate points on any $g$ and if there exists a function $A(x), x \in \mathbf{R}$ subject to the following conditions:

( $\alpha$ ) $A$ is positive continuous exceeding some constant $\lambda$ and becomes infinite with $x$.

( $\beta)\left|w\left(x_{1}\right)\right|+\left|w\left(x_{2}\right)\right|>A(x)$ for $x>\lambda$ and $-x_{1}$ and $x_{2}>x$.

(c) The function $A$ is independent of $g$.

In particular then, this hypothesis is fulfilled on all surfaces of negative curvature and can also hold on surfaces having regions of positive curvature.

The methods of this paper depend heavily on the paper [4] of 1924, which in turn explores the ideas we encountered in the Morse Trajectory paper, but now in the context of closed surfaces of genus $>1$. On an arbitrary such surface one of course cannot expect a purely combinatorial description of all geodesics. On the other hand Morse shows that certain geodesics-which he calls of class $A$-behave very much like the geodesics in the constant negative curvature case.

A geodesic $g$ is of class $A$ if any segment of $g$, i.e. $g \mid[a, b]$, minimizes the distance between its endpoints, amongst curves in its homotopy class.

To every such geodesic $g$ on $M$ Morse assigns a geodesic $g^{*}$ in the constant curvature model of $M$ by the following strategem.

Because the genus of $M$ is $>1$, its universal cover $\tilde{M}$ can be identified with the unit disc $|z|<1$ in the complex plane. Thus we may think of $\tilde{M}$ as this disc in some Riemann structure which is invariant under the action of $\pi_{1}(M)$ acting as a subgroup of $S L(2, \mathbf{R})$ on $|z|<1$. Now then every $g$ on $M$ lifts to a curve $\tilde{g}$ on $\tilde{M}$, and Morse shows that there is at least one non-Euclidean straight line $g^{*}$ on $|z|<1$, such that $\tilde{g}$ is in a finite neighborhood of $g^{*}$ and vice versa. Further he shows that this correspondence is one-to-one under the hypothesis of uniform instability.

The ideas and insights of these papers are clearly precursors of the great new developments in this field in the 60's, due in large part to Anosov, Arnold, Sinai, Smale and others. In particular the condition of uniform instability is a precursor of the notion of an Anosov flow. See [S4] and [S5] for instance.

\footnotetext{
${ }^{3}$ Warning: This notion should not be confused with structural instability of a flow!
} 
4. The Morse theory. In 1925 Marston Morse published his first paper concerned with the distribution of critical points of a function [5]. He returned to this subject in one form or another for the rest of his career, and there are at least fifty papers in his bibliography presenting different settings of this theory.

The paper [5] discusses the finite-dimensional case, but right away for manifolds with boundary, so that the famous "Morse inequalities" appear here less symmetrically than in later versions. Because these inequalities play such a natural role in contemporary mathematics, let me formulate them and outline their proof here in contemporary language, and only then return to the subject of Morse's methods in these early papers.

4.1. The Morse inequalities. Let $f$ be a smooth function on a smooth manifold $M$. A critical point of $f$ is then a point $p$ at which

$$
\left.\frac{\partial f}{\partial x^{i}}\right|_{p}=0
$$

relative to some local system of coordinates $\left(x^{1}, \ldots, x^{n}\right)$. At such a point the matrix

$$
H_{p} f=\left.\frac{\partial^{2} f}{\partial x^{i} \partial x^{j}}\right|_{p}
$$

has two intrinsic invariants which can be taken to be the rank and the number of negative eigenvalues. Morse calls the corank of $H_{p} f$ the nullity $n(p)$ of $p$ (as a critical point of $f$ ) and the number of negative eigenvalues the index of $p$. This index is usually denoted by $\lambda_{p}$. Clearly it represents the dimension of the largest subspace on which the quadratic form $H_{p} f$ is negative definite.

With these concepts understood, Morse calls $f$ a nondegenerate function if all of its critical points are nondegenerate, and a first quite general result concerning such functions is that on a compact $M$ a nondegenerate function can only have a finite number of critical points. One may therefore count the critical points of a fixed index $k$, to obtain an integer $m_{k}(f)$ or simply $m_{k}$, which Morse calls the $k$ th type number of $f$.

Some of us also like to introduce the polynomial

$$
\Re_{t}(f) \equiv \sum t^{k} m_{k} \equiv \sum_{p} t^{\lambda}
$$

and refer to it as the "Morse polynomial" of $f$.

This terminology recalls another famous polynomial in topology, the Poincaré polynomial

$$
P_{t}(M)=\sum \operatorname{dim} H_{i}(M) t^{i}
$$

where $H_{i}$ denotes the $i$ th homology group of $M$ with coefficients in some field, and Morse's insight is now expressed by the following theorem, which essentially determines a lower bound for $\mathfrak{K}_{t}(f)$ in terms of $P_{t}(M)$.

THEOREM. If $f$ is any nondegenerate function on the compact $n$-manifold $M$, then its type numbers $m_{k}$ and the Betti numbers $b_{k}=\operatorname{dim} H^{k}(M)$ of $M$ satisfy 
the inequalities

$$
\begin{aligned}
m_{0} & >b_{0}, \\
m_{0}-m_{1} & <b_{0}-b_{1}, \\
m_{0}-m_{1}+m_{2} & >b_{0}-b_{1}+b_{2}, \\
\cdots & \cdots \\
m_{0}-m_{1}+\cdots+(-1)^{n} m_{n} & =b_{0}-b_{1}+\cdots+(-1)^{n} b_{n} .
\end{aligned}
$$

These then are the famous "Morse inequalities." They clearly set bounds for the $m_{k}$ 's in terms of the $b_{k}$ 's. Indeed subtracting successive lines of (4.5) yields

$$
m_{k}>b_{k} \text {. }
$$

But (4.5) is of course stronger. Note also the equality of the last line.

All in all one should think of these inequalities as a beautiful extension of the minimum principle. Indeed in the present context this principle simply asserts that $m_{0}>b_{0}$.

The inspiration for Morse's work was the minimax principle of G. D. Birkhoff, which occurs in the middle of Birkhoff's famous paper on dynamical systems of two degrees of freedom, [B1] and there amounted to the inequality

$$
m_{0}-m_{1} \leqslant b_{0}-b_{1}
$$

on 2-surfaces.

Indeed on p. 346 of the paper [5] under discussion, Morse quotes the previous inequality and then writes: "Upon reading Birkhoff's paper it occurred to the author that inasmuch as there are $(n+1)$ different kinds of critical points possible (in a sense to be defined later) there ought to be relations analogous to (4.7) ...."

Morse's method however is quite different from Birkhoff's, and it was only in the 30's that Birkhoff-in collaboration with Hestenes [B3]-produced a proof of the Morse inequalities along the lines of the minimax principle.

In his book [C.V.], Morse also mentions that the equality in the last line of (4.5) already occurs in Poincaré. The general equality seems to have been discovered quite independently-and indeed from quite different points of view-at about the same time by $\mathrm{H}$. Hopf, M. Morse and S. Lefschetz.

The method of proof which led Morse to the result is the one we still use today, and it naturally falls into two quite distinct parts. Part 1 is purely geometric, and is essentially based on the principle of deforming $M$ along the directions of steepest descent for $f$. These arguments culminate in the following two theorems.

THEOREM A. Suppose that our nondegenerate $f$ has no critical points in the region $a<f<b$. Then if $M_{t}$ denotes the "half space" where $f<t$ on $M$, there is a diffeomorphism of $M_{a}$ with $M_{b}$

$$
M_{b} \simeq M_{a} .
$$

This result (Morse's Lemma 6, p. 359) follows pretty directly from "pushing $M_{b}$ into $M_{a}$ along the gradient of $f$ " and of course uses the compactness of the $M_{t}$ 's. 
The next result on the other hand, really goes to the heart of the matter of what happens to $M_{t}$ as $t$ passes a critical value. To explain it in contemporary language let me remind the reader of the concept of attaching a thickened cell to a manifold. The underlying geometric idea is best gleaned from the following diagram

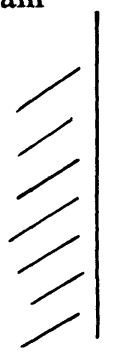

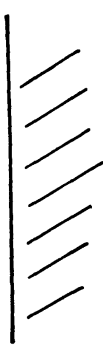

$\boldsymbol{X}$

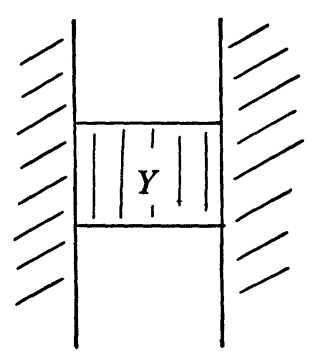

$X \cup Y$

Figure 2

where we have attached the "thickened 1-cell $Y$ " to $X$. Here $X=\{(x, y)|| x \mid$ $>1\} ; Y$ is the square $I \times I$, given by $|x|<1,|y|<1$, and the terminology arises from the fact that homotopically $X \cup Y$ is quite equivalent to the space

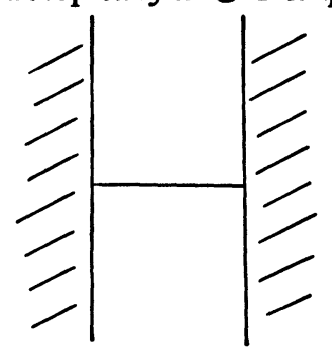

FIGURE 3

where $I$ is the interval $|x|<1$ on the $X$-axis. Thus as far as the glueing of part of the boundary of $Y$ into the boundary of $X$, the two factors of $Y$ play quite distinct roles, i.e. the second one just plays the role of a "thickening". Quite generally one now says that $X^{\prime}$ is obtained from $X$ by attaching a thickened $k$-cell, if $X^{\prime}$ is obtained from the disjoint union

$$
X^{\prime}=X \amalg e^{k} \times e^{n-k}
$$

by glueing "half" the boundary, $\partial e^{k} \times e^{n-k}$, of the cell $e^{k} \times e^{n-k}$ into the boundary $\partial X$ by a diffeomorphism

$$
\alpha: \partial e^{k} \times e^{n-k} \rightarrow \partial X .
$$

(The resulting manifold is then also often denoted by $X \cup_{\alpha} e^{k} \times e^{n-k}$.)

With all this understood we come now to the fundamental theorem of the Morse theory.

THEOREM B. Suppose that $f$ has only one nondegenerate critical point $p$, of index $\lambda$ in the range $a<f<b$ and that $a<f(p)<b$. Then

$$
M_{b} \simeq M_{a} \cup_{\alpha} e^{\lambda} \times e^{n-\lambda} ;
$$

that is, $M_{b}$ is diffeomorphic to $M_{a}$ with a thickened $\lambda$-cell attached. 
This theorem summarizes-in modern terminology-the Lemmas 7, 8, 9, 10 of [5], and the figure which follows should make the theorem plausible. Here we have drawn the behavior of $M_{b}$ relative to $M_{a}$ in a 2-dimensional example, near a critical point $p$ of $f$, in terms of a coordinate system $x, y$, such that, near $p$,

$$
f=f(p)+y^{2}-x^{2} .
$$

The so-called Morse Lemma assures one that such coordinates always exist near a nondegenerate critical point of index 1 . In fact this lemma asserts that near a nondegenerate critical point of index $\lambda$, one can always find coordinates $\left(x_{1} \ldots x_{\lambda}, y_{1} \ldots y_{n-\lambda}\right)$ such that

$$
f=f(p)+\sum_{i=1}^{n-\lambda} y_{1}^{2}-\sum_{j=1}^{\lambda} x_{j}^{2} \text { near } p \text {. }
$$

Now then, in such a coordinate patch $U$, and taking $a$ and $b$ equal to $f(p)-\varepsilon$ and $f(p)+\varepsilon$ respectively with $\varepsilon$ small and positive, one clearly finds that $\partial M_{b}$-the boundary of $M_{b}$-intersects $U$ in the upper and lower arcs of the hyperbola

$$
y^{2}-x^{2}=\varepsilon>0
$$

while $M_{a}$ appears in $U$ as the shaded region. Finally consider the cross hatched region $Y$. It is clearly a thickened 1-handle attached to the closure of $M_{b}-Y$. On the other hand, the gradient flow can be used to "push" this $M_{b}-Y$ into $M_{a}$ and in fact to construct a diffeomorphism of $M_{b}-Y$ and $M_{a}$. Q.E.D.

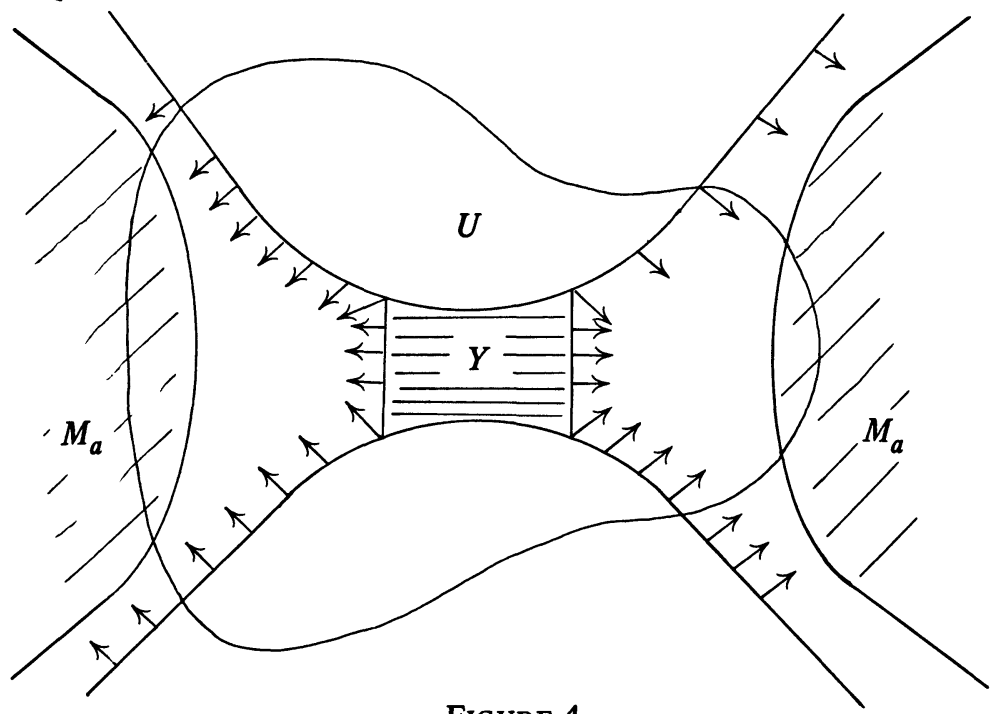

FIGURE 4

Now in 1980, the Morse inequalities follow from Theorems A and B by absolutely standard procedures of algebraic topology.

Precisely, one considers the sequence of maps

$$
M_{a} \hookrightarrow M_{b} \rightarrow M_{p} / M_{a}
$$


where in the last place we mean the space obtained from $M_{b}$ by collapsing $M_{a}$ to a point. From our thickened handle construction, that is Theorem B, it follows that

$$
M_{b} / M_{a} \simeq S^{\lambda}
$$

in the sense of homotopy. Here $S^{\lambda}$ of course denotes a $\lambda$-sphere. On the other hand, two of the basic properties of any homology theory are that

(A) They are invariant under homotopy equivalences.

(B) For any inclusion $X \subset Y$ of reasonable spaces there is induced a "long exact sequence"

$$
\rightarrow H_{k+1}(Y / X) \stackrel{\text { a }}{\rightarrow} H_{k}(X) \rightarrow H_{k}(Y) \rightarrow H_{k}(X / Y) \stackrel{\text { ’ }}{\rightarrow}
$$

I will not describe this notion in detail here; however in our situation this exactness immediately implies that

$$
\Delta P_{t} \equiv P_{t}\left(M_{b}\right)-P_{t}\left(M_{a}\right)=\left\{\begin{array}{l}
t^{\lambda} \\
\text { or } \\
-t^{\lambda-1}
\end{array}\right.
$$

On the other hand the corresponding change $\Delta \mathscr{N}_{t}$ in the Morse-series is clearly given by $t^{\lambda}$. Thus

$$
\Delta \mathscr{N}_{t}-\Delta P_{t}=\left\{\begin{array}{l}
0 \\
\text { or } \\
t^{\lambda-1}(1+t) .
\end{array}\right.
$$

Proceeding inductively we see that there exists a polynomial $Q(t)$ with nonnegative coefficients

$$
Q(t)=q_{0}+q_{1} t+\cdots, \quad q_{i}>0,
$$

such that

$$
\mathscr{K}_{t}(M)-P_{t}(M)=(1+t) Q(t) .
$$

But the inequalities $q_{i}>0$ in conjunction with (3.19) are seen to be precisely the "Morse inequalities". Q.E.D.

REMARKS. The account given in [5] of this homological part is of course couched by Morse in the language of the Analysis Situs of that time; that is of Veblen's fundamental book. From our perspective this essentially amounts to an informal sort of singular theory. There was no formalization of exactness about yet, but all the fine topologists of the era of course used all the exactness properties at various stages of their proofs. Essentially then it is fair to say that Morse's arguments in [5] already establish Theorem A and the homological consequences of Theorem B in the language of his time. On the other hand in the precise formulation that I have given, that is, in the category of diffeomophisms, Theorem B only appears in Smale's work in the 60's, and to establish Theorem B in this precision considerable care has to be taken with concepts such as smoothing corners, etc. In the 50's René Thom, E. Pitcher and I used to formulate this Theorem B in purely homotopy-theoretic terms-that is in the form

$$
M_{b}=M_{a} \cup_{\alpha} e_{\lambda}
$$


expressing the fact that as far as homotopy theory is concerned one simply attaches a $\lambda$ cell as one passes a critical point of index $\lambda$.

Smale's proper understanding of the Morse theory in the diffeomorphism category of course was the first step in his handle-body theory, and finally in his fundamental contributions to differential topology. (See [S1], [S3] for instance.)

Before proceeding to the infinite-dimensional settings of the theory, which really was the driving force in Morse's work throughout, let me show off the power of these inequalities in one or two examples.

First of all let me record the "Lacunary Principle" of Morse, which follows directly from the $(1+t)$ factor on the right to (3.19).

LACUNARY PRINCIPLe. If in $\mathfrak{M}_{t}(f)$ all products of two consecutive coefficients vanish, i.e. $m_{j} \cdot m_{j+1} \equiv 0$, then

$$
\mathfrak{M}_{t}(f)=P_{t}(M)
$$

for any coefficients field.

Thus for such a function $\mathfrak{T}_{t}(f)$ computes the Poincaré Polynomial of $M$-and as a consequence the space $M$ is even "torsion-free". We call functions satisfying (4.21) perfect Morse functions, and they clearly furnish us with a convenient way of computing $P_{t}(M)$. As an example consider the complex projective space $P(V)$ of one-dimensional subspaces of a finite-dimensional vector space.

Given a hermitian form $(H x, x)$ on $V$, it can be used to define the function

$$
\tilde{f}(x)=(H x, x) /(x, x)
$$

on the unit sphere $S(V)$, of $V$, and as $f(\lambda x)=f(x)$ for all $\lambda \neq 0$ in the complex number field $\mathbf{C}$, this $\tilde{f}$ induces a function $f$ on $P(V)$. The critical points of $f$ are now easily seen to be given by the eigenspaces of $H$

$$
H x_{i}=\mu_{i} x_{i} \text {. }
$$

Hence if we assume, as we may, that the eigenvalues of $H$ are distinct, and that they are ordered in ascending order,

$$
\mu_{1}<\mu_{2}<\cdots<\mu_{n}, \quad n=\operatorname{dim} V,
$$

we see that $f$ will have precisely $n$-critical points. Furthermore they turn out to be nondegenerate and their indices are easily computed to be

$$
\lambda\left\{x_{i}\right\}=2(i-1) \text {. }
$$

(Indeed moving $x_{i}$ in the direction of the earlier $x_{j}$ clearly decreases $f$ quadratically, and as we are dealing in complex directions each of these contributes 2 to the index.) In short then

$$
\Re_{t}(f)=1+t^{2}+\cdots+t^{2(n-1)} \text {. }
$$

This is a lacunary series and therefore $f$ is perfect. Thus

$$
P_{t}\left(\mathbf{C} P_{n}\right)=1+t^{2}+\cdots+t^{2(n-1)}
$$

and $\mathrm{CP}_{n}$ is torsion-free. Q.E.D.

In this and similar examples one may thus turn the Morse theory around so to speak and use it as a computational tool. This was often done by Morse in 
his subsequent work. For instance in his book written in 1934 [C.V.] he computes the mod 2 cohomology of the symmetric product of two spheres in this manner. Still, he left many examples where the Morse theory, used similarly, easily produces results which seem quite inaccessible by other means. Let me just mention two. The first, which I noticed in 1951 (see [B6]) is in a sense a generalization of the complex projective space example we just discussed. The theorem asserts that wherever $M$ is the orbit of a point under the adjoint representative of a compact Lie group $G$ on its Lie algebra $\mathfrak{g}$, then the distance function $f_{P}$ from a generic $p \in \mathfrak{g}$ to $M$ is perfect because its Morse series is a function of $t^{2}$ alone and hence lacunary. Thus these orbits are all free of torsion and the Morse theory leads to a description of their homology in terms of the usual paraphernalia of Lie group theory, i.e. diagrams, Weyl groups, etc.

A second-and in my mind maybe the most striking application of this procedure-is to the Lefschetz Hyperplane Theorem in algebraic geometry. Various ways of deducing this fundamental result were found in the 50's and finally a beautifully simple proof of the Lefschetz theorem was given by Andreotti and Frankel [A]. Here, as really also in my examples, the complex variable situation rather naturally forces special properties on the indices of suitably natural functions on the spaces in question. In my case they all turned out to be even; in the Lefschetz theorem they all could be bounded. Via the Morse theory these easy local computations then turn out to have global topological implications.

4.2. The calculus of variation setting of the Morse Theory. Beautiful as the considerations of the previous sections are, it is clear that to Morse they were mainly "results along the way" to his real goal-a corresponding theory in the calculus of variations. For instance while he was writing the paper discussed previously, he had already lectured the Society on his results in this infinitedimensional context.

The situation envisaged by Morse is the following one. There is first of all an underlying manifold $M$, and on it a fixed variational form $F(q, \dot{q})$, which defines a "functional"

$$
J(u)=\int_{a}^{b} F(u, \dot{u}) d t
$$

on the space of piecewise differentiable paths $u$ on $M$. In modern terminology $F$ is of course a function on the tangent bundle of $M$, and it is understood that it satisfies the usual nondegeneracy condition

$$
\left\{\frac{\partial^{2} F}{\partial \dot{q}^{i} \partial \dot{q}^{j}}\right\}>0 .
$$

Now, given a set of "admissible boundary conditions" Morse restricts himself to the space $\Omega$ of those paths which satisfy the condition in question and develops the "Morse-theory" for the function $J$ on $\Omega$. That is, he succeeds in defining the notion of index and nullity for any extremal in $\Omega$, and in proving that for any nondegenerate $J$ (i.e. all extremals of $J$ in $\Omega$ have nullity zero) the Morse inequalities persist.

With hindsight, and fifty years of experience, it is of course now pretty 
clear what to expect. The "tangent space" to an extremal, $s$, is the set of vector fields along it, subject to certain boundary conditions, so that the Hessian of $J$ at such a critical segment should be the quadratic form on this space furnished by the second variation of $J$.

Once a framing of the tangent-space to $M$ along $s$ is chosen, the space of vector-fields along $s$ is simply the space of $\mathbf{R}^{n}$-valued functions of $t$-the parameter along $s$-satisfying certain boundary conditions at the endpoints. Finally after integration by parts, this Hessian should take the form

$$
H_{s} J(x, x)=\int_{a}^{b}(L x, x) d t, \quad a \leqslant t \leqslant b,
$$

where $x(t)$ represents the tangent field, and $L$ is a linear second order differential operator

$$
L=A(t) d^{2} / d t^{2}+B(t) d / d t+C(t) .
$$

It follows that the eigenvalue problem

$$
L x=\lambda x, \quad x \in \beta(s),
$$

with $x(t)$ subject to the boundary conditions $\beta(s)$, is well posed and hence has only a finite number of independent solutions with $\lambda \leqslant$ some constant.

Thus Morse defines the index and nullity of an extremal $s$ by

$$
\begin{aligned}
\operatorname{nullity}(s) & =\operatorname{dim} \text { of solutions of } L x=0, \\
\operatorname{index}(s) & =\operatorname{dim} \text { of solutions to } L x=\lambda(s), \\
& x \in \beta(s) \text { with } \lambda<0
\end{aligned}
$$

and with this definition every nondegenerate $J$ should have a well-defined Morse-series

$$
\mathfrak{M}_{t}(J)=\sum_{s} t^{\lambda(s)}
$$

where $s$ runs over the extremals of $J$ in $\Omega$ and $\lambda(s)$ denotes the index.

I say series, because now there is of course no a priori bound on $\lambda(s)$ as there was in the finite-dimensional case. There remains the question however, whether this series is a well-defined formal power series; for conceivably there might be an infinite number of extremals of fixed index. However, as Morse shows, under appropriate completeness conditions this cannot happen on any component of $\Omega_{0}$ of $\Omega$, and then the coefficients of the restricted Morse series

$$
\mathscr{T}_{t}^{0}(J)=\sum t^{\lambda(s)}, \quad s \in \Omega_{0}
$$

satisfy the Morse inequalities relative to the Poincaré Series of $\Omega_{0}$

$$
P_{t}\left(\Omega_{0}\right)=\sum t^{i} \operatorname{dim} H^{i}\left(\Omega_{0}\right)
$$

Let me illustrate the situation with an example which is certainly of the greatest geometric significance. For our functional $J$ we take the energy-function on a complete Riemann manifold $M$

$$
J(u)=\int_{0}^{1}|\dot{u}|^{2} d t .
$$


For simplicity our space $\Omega$ will be taken to be the space of piecewise differentiable curves parametrized by a parameter $0 \leqslant t \leqslant 1$ proportional to arc-length, and the boundary conditon we impose on $\Omega$ is the fixed endpoint condition

$$
u(0)=p, \quad u(1)=q, \quad p, q \in M .
$$

The eigenvalue problem associated to a given extremal $s$, in this situation is then invariably described by

$$
-\left\{\nabla_{X}^{2} \cdot Y+R(X, Y) X\right\}=\lambda Y, \quad Y_{0}=Y_{1}=0,
$$

where $Y$ is a normal vector field along $s, X$ is the tangent field along $s, \nabla_{X}$ is the Levi-Civita invariant derivative along $X$ and $R(X, Y)$ the curvature of the Riemann structure.

In Riemannian geometry one calls the vector-fields $Y$ along $s$ subject to

$$
\nabla_{X}^{2} Y+R(X, Y) X=0
$$

the space of Jacobi-fields along $s$, and in terms of them two points $a$ and $b$ on $s$ are called conjugate along $s$, if and only if there is a Jacobi field $Y \neq 0$, which vanishes at $a$ and $b$. The multiplicity of such a conjugate pair is then the dimension of the subspace of Jacobi-fields which vanish at $a$ and $b$.

In view of (4.39), we see then that the critical segment $s$ in $\Omega$ has nullity 0 if and only if the endpoints of $s$ are not conjugate along $s$. Conjugate points are of course of great geometric interest and also often intuitively apparent. Thus if $s$ can be embedded in a " $k$-parameter family" $s(\alpha)$ of geodesics, all having the same endpoints as $s$, then these endpoints are conjugate along $s$ to order at least $k$. This follows from Jacobi's principle that the vector fields $\partial s_{\alpha} /\left.\partial \alpha\right|_{\alpha=0}$ along $s$ satisfy the Jacobi equation whenever $s_{\alpha}$ is a parameter family of extremals reducing to $s$ at $\alpha=0$.

For instance on the unit sphere $S^{n}$ in $\mathbf{R}^{n+1}$, only antipodal points are conjugate and the multiplicity of an antipode pair $(p, \bar{p})$ is $(n-1)$, independently of the segment s joining them.

This follows from the great symmetry of the sphere. Indeed, every geodesic on $\boldsymbol{S}^{\boldsymbol{n}}$ must lie in the two-plane spanned by its initial point and direction, and so is simply an arc along a great circle $S^{n}$. Furthermore, as the curvature of $S^{n}$ is 1 , the Jacobi equations simply take the form

$$
\left(d^{2} / d t^{2}\right) X(t)+k^{2} X(t)=0
$$

$k$ being the length of the segment $s$ under consideration. (Recall our parametrization convention in $\Omega$.)

Thus the nullity of a segment $s$ is always 0 unless $s$ has length a multiple of $\pi$, and then its endpoints are clearly antipodal or equal.

Finally the nullity of an $s$ with antipodal endpoints $(p, \bar{p})$ is $(n-1)$ in conformity with the fact that all the geodesics through $p$ pass through $\bar{p}$.

It is now a simple matter to compute the Morse series appropriate to this example. Indeed the number of negative eigenvalues of (4.41) are also quite easily estimated, and one then finds the following state of affairs. First of all let us survey the totality of geodesics joining the north pole $p$ say, to the point 
$q$, one quarter of the great circle away. Thus we start with the figure

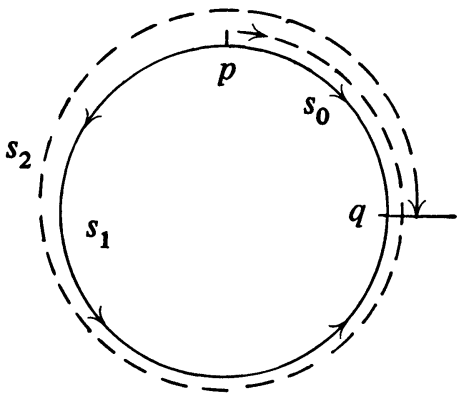

FIgURE 5

where we have also indicated the first three extremals joining $p$ to $q$. Notice that if one "unfurls" the circle, i.e. passes to its universal cover $\mathbf{R}$, one obtains the following overview of the situation

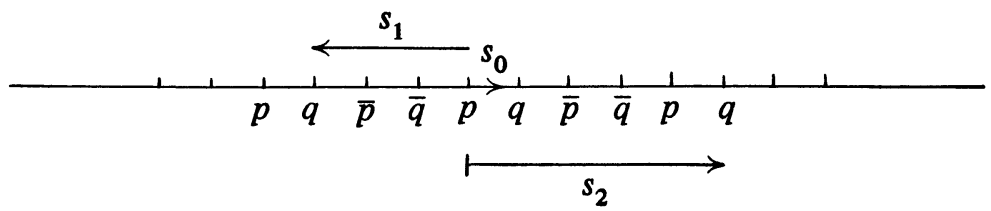

Figure 6

where the bar denotes antipodes.

In short then the geodesic segments in $\Omega$ correspond to the line-segments in $\mathbf{R}$ joining the origin to the lattice of integer points $\boldsymbol{n}$ on $\mathbf{R}$ which are congruent to $1 \bmod 4$.

Now then if we estimate the index of each $s_{k}$ we find that

$$
\text { index } s_{k}=|k|(n-1)
$$

so that the Morse Series for this situation takes the form

$$
\Re_{t}(J)=\sum_{k=0}^{\infty} t^{k(n-1)} \equiv \frac{1}{1-t^{n-1}} .
$$

Note that this series is lacunary for $n>2$, so that the Morse inequalities become equalities and yield the formula

$$
P_{t}(\Omega)=\frac{1}{1-t^{n-1}}
$$

in that range. Actually this equality also holds for $n=2$, but for that one needs a more geometric argument.

Morse was in possession of this formula in the early thirties and most probably in the late twenties. (It certainly occurs in his book [C.V.],-rather buried-as Theorem 15.1 on p. 247, but I find no reference to it earlier.)

Morse did not seem to attach particular interest to this computation except insofar as it enabled him to prove the theorem I already mentioned in the introduction. That is: For any Riemann structure on $S^{n}$, "there must be an 
infinite number of geodesics $g_{1}, g_{2}, \ldots$ joining any two fixed points $A_{1}$ and $A_{2}$. The length of $g_{n}$ and the number of conjugate points of $A_{1}$ on $g_{n}$ become infinite with n".

The part of this statement in quotation marks is verbatim from the corollary on p. 248 of [C.V.]. To understand it properly two essentially new points have to be added to our discussion. The first of these is the generic nature of the nondegnerate case. Morse was aware of what we nowadays call the Sard Lemma, although in slightly different technical settings, and it is an understatement to say that he preferred it to be called the Morse-Sard Lemma. Thus, to proceed to the prior corollary from the computations of $P_{t}(\Omega)$ he first approximates $A_{1}$ and $A_{2}$ by a sequence $\left(A_{1}^{n}, A_{2}^{n}\right)$ of generic points-i.e. for which the energy function is nondegenerate. For these the Morse inequalities clearly imply the existence of geodesics $\left\{g_{n}\right\}$ with index tending to $\infty$, and as nondegenerate critical points are isolated, with length also tending to $\infty$. Morse then argues by continuity to establish that a sequence $\left\{g_{n}\right\}$ with index and length tending to $\infty$ exists in the limiting case also.

The second point to be added is now the beautiful index-theorem of Morse which in the present context is given by

The index of an extremal $s$ in the fixed endpoint problem is equal to the number of conjugate points of one endpoint in the interior of $s$.

Thus if the index of $g_{n}$ tends to infinity so do the number of conjugate points of one endpoint in the interior of $g_{n}$. With the aid of this result the path to the corollary under discussion should now be clear.

Note that this index theorem also enables one to read off the index formula (4.42) from Figure 5: The index of $s_{1}$ is equal to $(n-1)$ times the number of $\bar{p}$ points on it.

For a general symmetric space there is, by the way, a completely analogous description of the geodesics joining two points. Again they correspond to segments joining the origin to a lattice-this time in $\mathbf{R}^{k}$ where $k$ is the rank of the space, and the index of a segment $s$ can be read off from the number of times $s$ pierces a certain family of hyperplanes. See [B8].

This index theorem fits, properly speaking, into a natural extension of the classical Sturm theory for the differential equation

$$
-\left(x^{\prime \prime}+q x\right)=\lambda x
$$

and coming at it from the calculus of variations as he did led Morse to redo the classical theory as well as extend it in a variety of ways along quite new lines.

A thorough account of this work is to be found in his book [V.A.] called Variational Analysis.

The point is that in all properly posed variational problems there is some type of index theorem; however for some boundary conditions the answer is more difficult and less satisfying. Let me illustrate. If we consider the $J$ of our discussion on the space $\Omega(p, N)$ of paths starting on a submanifold $N$ and ending at a point $p$, then the index theorem in this context becomes what Morse calls the focal-point theorem:

The index of an extremal $s$ is equal to the number of focal points of $N$ in the interior of $s$. 
The following figure illustrates this theorem clearly

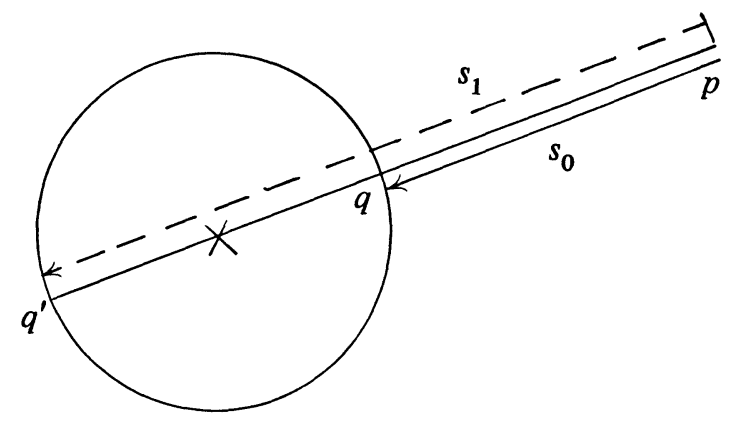

FIGURE 7

Here the underlying manifold is the plane and $N$ is the unit circle, while $p$ is taken as a point not on the circle. The extremals of $J$ on $\Omega(N, p)$ are geodesic segments starting perpendicularly on $N$ and joining $N$ to $p$. Here they are therefore given by the two straight lines $s_{0}$ and $s_{1}$ joining $q$ and $q^{\prime}$ to $P$. The only focal point of $N$ is the center of the circle and it has multiplicity 1. Thus index $s_{1}=1$. Note that the same picture is valid for the $n$-space in $\mathbf{R}^{n}$ but that then $s_{1}$ would have index $(n-1)$ corresponding to the fact that the origin is a focal point of multiplicity $(n-1)$.

However, for more complicated situations, for instance when we deal with two end manifolds $N_{1}$ and $N_{2}$ both of dimensions greater than 0 , or with periodic boundary conditions

$$
u(1)=u(a), \quad \dot{u}(1)=\dot{u}(0),
$$

the index of an extremal cannot be read off so simply from the behavior of the solutions to the Jacobi equation along the extremal.

This whole subject is still of great interest and has been taken up by a variety of authors (see [K]). Basic in all of them is however Morse's insight that this index has a topological meaning which can be computed by an intersection-number.

REMARKS. Inadequate as the previous account of what one might call the concrete "Morse Theory" is, it will have to do in the present context. It is meant more as an excursion into his work; and to a certain extent as a biased excursion into that part of the theory which I fell in love with thirty years ago. It is also the part of the theory which has had the most direct bearing on homotopy theory. My first remark is therefore devoted to this connection.

(1) The space $\Omega$ of paths joining two points on $M$ is nowadays called the Loop Space of $S$ and denoted by $\Omega M$. Its homotopy type is independent of the points chosen (as was already shown by Morse), and it plays a vital role in all of homotopy theory. In fact it appeared right away in the first papers of Hurewitz on the higher homotopy groups through its characteristic property

$$
\pi_{k}(\Omega M)=\pi_{k+1}(M)
$$

Of course the homotopy theorists and Morse each went their own way-essentially until R. Thom and I realized what a powerful tool the Morse theory 
could be in this context. For instance our discussion of the critical points of $J$ on $\Omega S^{n}$ immediately implies a formula of the type

$$
\Omega S^{n}=S^{n-1} \cup_{\alpha} e_{2(n-1)} \cup_{\alpha} e_{3(n-1)} \cdots
$$

That is, $\Omega S^{n}$ is built up from the $(n-1)$ sphere by attaching cells of higher and higher dimension. On the other hand an elementary result of homotopy theory is that attaching a $k$-cell does not effect the homotopy groups in dimension $<k-2$. Thus (4.47) and (4.48) together imply that

$$
\pi_{k+1}\left(S^{n}\right)=\pi_{k}\left(\Omega S^{n}\right)=\pi_{k}\left(S^{n-1}\right), \quad k<n-3 .
$$

This is the "Freudenthal stability theorem" for the homotopy of the spheres.

In the later fifties I finally realized that what Morse had done for the spheres worked even more smoothly for the compact groups and some of their homogeneous spaces. For instance, if $U_{n}$ is the $n$th Unitary group, the correct analogue of (4.48) is given by two formulas

$$
\begin{cases}\Omega U_{2 n}=U_{2 n} / U_{n} \times U_{n} \cup_{\alpha} e_{1} \cup_{\alpha} e_{2} \ldots, & \operatorname{dim} e_{i}>n, \\ \Omega U_{2 n} / U_{n} \times U_{n}=U_{n} \cup_{\alpha} e_{1} \cup e_{2} \cup \ldots, & \operatorname{dim} e_{i}>n .\end{cases}
$$

Combined with the known fact that $\pi_{k}\left(U_{n}\right)$ is also "stable" in the sense that it does not depend on $\boldsymbol{n}$ for $\boldsymbol{n}$ large compared to $\boldsymbol{k}$, these relations immediately lead to the periodicity $\pi_{k}(U)=\pi_{k+2}(U)$ with $\pi_{k}(U)$ the stable value of $\pi_{k}\left(U_{n}\right)$. In this framework [B5], eight such formulas finally lead to the 8-fold periodicity $\pi_{k}(0) \simeq \pi_{k+8}(0)$ of the stable orthogonal group.

(2) My next remark deals with the techniques Morse uses in his papers and his book. Basically he deals with the infinite-dimensional function space by deforming compact sets in it into a finite-dimensional space of broken extremals, and then applying his finite-dimensional results.

Apart from technicalities-which actually can become quite formidablethis is an approach some of us still favor. Others, like Smale-Palais [P], [S3], have cast the whole theory in terms of infinite-dimensional Hilbert-manifolds from the very outset. In such an account the space $\Omega$ of course has to be topologized in a slightly different way than in Morse's approach. Morse essentially endowed $\Omega$ with a metric topology, with metric

$$
d\left(g_{1}, g_{2}\right)=\sup _{t}\left|g_{1}(t), g_{2}(t)\right|+J\left(g_{1}\right)-J\left(g_{2}\right) \text {. }
$$

Topoiogists think of $\Omega$ as simply the space of continuous maps in the compact open topology. Luckily all these topologies yield the same "weak homotopy type" for $\Omega$, so that the same Morse inequalities emerge at the end. I should confess here also that Morse did not work with the energy integral, but always with the parameter-free length integral

$$
l(u)=\int_{a}^{b}|\dot{u}| d t
$$

or its more general analogue. This involved him in considerable technical difficulties which, I should add, he overcomes with tremendous ingenuity and geometric insight.

Altogether Morse's geometric power is phenomenal and he seems to have been able to compute anything he set his mind to. For instance, he computes the homology of the symmetric product of the $n$-sphere by hand, so to speak. 
That is, he constructs the explicit cycles and thus in 1930 he really anticipates the work of P. A. Smith and Richardson on the one hand, and that of Steenrod in the 50's on the other.

Morse's sheer power is however best exhibited in his attack on the closed geodesics problem. These closed geodesics can be thought of as extremals of our $J$ considered on the space of all piecewise smooth paths $\Gamma$ subject to $u(0)=u(1)$. However for this problem there is no "nondegenerate case" for, with a given $u$, all its translates are also critical. Thus in the "most nondegenerate situation" one can hope for one still has to deal with critical sets of circles. Furthermore each genuine geometric closed geodesic gives rise to an infinite number of such critical circles corresponding to the number of times one circumnavigates it. Thus it is hard to estimate the number of closed geodesics on $S^{n}$ say, in terms of the topology of $\Gamma S^{n}$. Still one can, in this manner, easily deduce that there is at least one nontrivial closed geodesic on $S^{n}$ in any Riemann structure. On the other hand Morse was shooting for more, and so, to my knowledge, he never bothered to compute the homology of $\Gamma S^{n}$. Rather, he felt that because $J$ was naturally invariant under translations, the correct space on which $J$ was to be considered was in some sense the quotient of $\Gamma S^{n}$ by the action of $S^{1}$ on $\Gamma$, given by $u(x) \rightarrow u(x+\theta)$, $0<\theta<1$.

Thus he made polygonal approximations to $\Lambda S^{n}$, divided them by the appropriate cyclic group actions and then attempted to pass to the direct limit (!) in homology. The Betti-numbers he obtained that way he called the "circular connectivities of $S^{n}$ ", and they were supposed to play the same role vis-à-vis the closed extremal problem which the Betti numbers of $\Omega M$ play vis-à-vis the fixed endpoint problem.

Unfortunately an error crept into this computation-an error, which by the way, I fell into myself in my paper [B4] trying to "modernize" Morse's computations-which was caught by L. Schwartz in 1957. This whole subject has had a lively history since then with many contributors-and also many subsequent mistakes. (See [K] for an excellent bibliography and account of this general topic. However, Klingenberg's main result is still in doubt at this time.) All in all the last word has not yet been written on it and I cannot help feeling that there remain many clues to be found in Morse's amazing last chapter to his Colloquium volume [C.V.].

4.3. Abstract settings of the Morse theory. In a series of papers [36], [44], [59], in the thirties, forties, and fifties, Morse explores various general formulations of his theory. In these papers he usually works on an abstract metric space $M$, with a function $F$ not necessarily continuous, and to obtain some analogue of the Morse inequalities in these situations he uses the Vietoris homology theory.

This is then the study of the homology behavior of the set $F<a$ under very general conditions. More precisely let

$$
H(a, b)=H(F<b, F<a), \quad a<b,
$$

denote the relative homology of the sets indicated. A critical value of $F$ is then defined by Morse as any number $c$, such that

$$
H\left(c, c^{-}\right)=\lim _{a \rightarrow c^{-}} H(c, a)
$$


is nontrivial, and the problem is now to relate the "sizes" of these critical $H\left(c, c^{-}\right)$to the "size" of $H(M)$. In a sense Morse was here grappling with ideas which were properly understood only 15 years later by Leray.

Indeed the general scheme of the Leray spectral sequence can be thought of in the following context. One is given a filtering

$$
M_{0} \subset M_{1} \subset \cdots M_{n}
$$

and asks how the groups $E_{1}=\bigoplus H\left(M_{i}, M_{i-1}\right)$ are related to $H(M)$. Leray's answer is of course that one can proceed in an orderly manner from $E_{1}$ to $E_{2} \ldots$ the passage from $E_{k}$ to $E_{k+1}$ always being a passage from a chain complex to its homology. Finally at $E_{\infty}$ one is close to $H(M)$. Now it is a fact that if all objects in a chain complex $A$ are finite dimensional, then the Morse inequalities pertain between the dimensions of the $A_{i}$ and those of $H\left(A_{i}\right)$. Thus the Morse inequalities already reflect a certain part of the "Spectral Sequence magic", and a modern and tremendously general account of Morse's work on rank and span in the framework of Leray's theory was developed by Deheuvels [D] in the 50's.

Unfortunately both Morse's and Deheuvel's papers are not easy reading. On the other hand there is no question in my mind that the papers [36] and [44] constitute another tour de force by Morse. Let me therefore illustrate rather than explain some of the ideas of the rank and span theory in a very simple and tame example.

In the figure which follows I have drawn a homeomorph of $M=S^{1}$ in the plane, and I will be studying the height function $F=y$ on $M$.
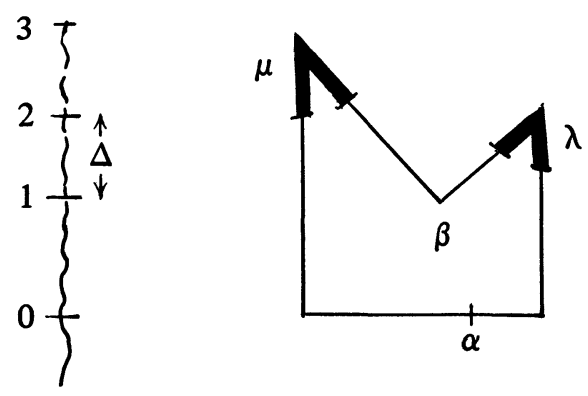

Figure 8

The values $a$ where $H\left(a, a^{-}\right) \neq 0$ are indicated on the left, and corresponding to each of these critical values a generator of $H\left(a, a^{-}\right)$is drawn on $M$, using the singular theory for simplicity. Morse calls such generators "caps". Thus $\alpha$ and $\beta$ are two "0-caps" and $\mu$ and $\lambda$ two "1-caps". Notice that every cap $u$ defines a definite boundary element $\partial u$ in

$$
H\left(a^{-}\right)=\lim _{\varepsilon \rightarrow 0^{+}} H(F<a-\varepsilon) ;
$$

Morse calls a cap $u$ linkable iff $\partial u=0$. Otherwise it is called nonlinkable.

In our example, $\alpha, \beta$ and $\mu$ are linkable while $\lambda$ is not.

Next Morse defines the span of a cap $u$ associated to the critical level $a$ in the following manner. 
Case I. $u$ linkable. Consider the inclusion homomorphism

$$
H\left(a, a^{-}\right) \stackrel{\iota_{b}}{\rightarrow} H\left(b, a^{-}\right), \quad b \geqslant a,
$$

and let $s(u)$ be the least upper bound of all $b$ with $\iota_{b} u \neq 0$. (If the set of such $b$ 's is unbounded $s(u)=\infty$.) The span of $u$ is now defined as

$$
\operatorname{span}(u)=s(u)-a \text {. }
$$

In our example $\alpha$ and $\mu$ have infinite span, while $\beta$ has span $\Delta$. Indeed the moment $b$ has passed (2) the cycle $\alpha$ can be deformed in $F \leqslant b$ into the region $F<F(\beta)$.

Case II. $u$ is not linkable. In this case consider $\partial u \in H\left(a^{-}\right)$and let $t(u)$ be the greatest lower bound of $b$ 's such that $\partial u$ is in the image of

Then set span $u=a-t(u)$.

$$
\iota_{b}: H(b) \rightarrow H\left(a^{-}\right), \quad b<a .
$$

In our example $\lambda$ is the only nonlinkable cap, and its span is now clearly again the number $\Delta$.

With these definitions understood Morse sets $m_{k}^{e}$ equal to the number of $k$-caps of span $>e$ and proves that under very general conditions these numbers will be finite for every $e>0$, and then obey the Morse inequalities.

For instance, in our example this amounts to $m_{0}^{e} \geqslant 1$ and $m_{0}^{e}=m_{1}^{e}$-for all $e>0$. And indeed, for $0<e<\Delta$ we have $m_{0}^{e}=2, m_{1}^{e}=2$, while for $e \geqslant \Delta$, $m_{0}^{e}=m_{1}^{e}=1$.

Our example is of course a very tame one; however, it should be clear that as one complicates it-for instance by introducing an infinite number of critical values clustering at some point, that as long as some sort of continuity is preserved, the number of $k$-caps of span $>e>0$ will be finite. On the other hand one then also sees that the Vietoris or Cech theory is the correct one in this framework.

5. Minimal surfaces. Marston Morse had developed the abstract setting of the variational theory which we just described in large part because he hoped to make it applicable to minimal surface theory and other variational problems. Unfortunately however a direct extension of the Morse Theory just does not work for variational problems in more than one variable. That is, if in analogy to the preceding discussion one attempts to study the area function, or its "energy", on the space $\Omega_{2}(M)$ of piecewise smooth maps of a disc into a Riemann manifold $M$ then the Morse theory will not work. In the context of the Palais-Smale theory [P], one understands this phenomenon in terms of the Sobolev inequalities, which show that the conditions on a map $\mu$ in $\Omega_{2}(M)$ to have finite area are far from forcing $\mu$ to be continuous. Thus, roughly, the space of continuous maps-which carries the topology-is too sparse in the space of admissible maps for a proper Morse theory to exist. (See $[P]$ in particular 16.)

Nevertheless in a series of papers, [40], [45], [47], [49] Morse and Tompkins do obtain applications of the Morse theory in this context. For instance in [40] they prove the existence of a minimal surface of nonminimum type, by applying the Morse inequalities to the following situation. Let $D$ be the unit disc $|z|<1$, and let $S=\partial D$ be its bounding circle. 
Now let $g: S \rightarrow \mathbf{R}^{n}$ be a simple closed curve in $\mathbf{R}^{n}$, satisfying a Lipschitz condition and parametrized by arc-length, i.e. defining an isometry of $S$ into $\mathbf{R}^{n}$. Next Morse and Tompkins introduced a space, $\Omega$, of "reparametrizations" of $g$

$$
g_{\varphi}(s)=g(\varphi(s))
$$

where $\varphi$ ranges over the homeomorphisms of $S^{1}$ which move points in only one direction, and keep three distinct points fixed. On this space they now consider the "Douglas-function"

$$
A(\varphi)=\frac{1}{16 \pi} \iint_{S^{\prime} \times S^{1}} \frac{\left|g_{\varphi}(\alpha)-g_{\varphi}(\beta)\right|^{2}}{\sin ^{2}\left(\frac{\alpha-\beta}{2}\right)} d \alpha d \beta
$$

and show that it satisfies the abstract conditions laid down in [32] by Morse. The geometric pertinence of $A$ is of course that $A(\varphi)$ is equal to the Dirichlet integral of the harmonic map $h_{\varphi}: D \rightarrow \mathbf{R}^{n}$ extending $g_{\varphi}$.

Now the space $\Omega$ is clearly contractible, so that in particular the Morse inequalities read

$$
m_{1}>m_{0}-1 \text {. }
$$

Morse and Tompkins cite an explicit $g$ with this property and so achieve their result.

In their other papers they explore this framework of questions in various directions, increasing the number of bounding circles, etc.

The paper [45] is by Morse alone, and is essentially analytic in character. There he gives an explicit form for the first variation of $A$ in the case of $n$-bounding contours, and shows that, if this variation vanishes, then the harmonic surface defined by the boundary conditions is indeed minimal.

6. Topological methods in the theory of a complex variable. In the middle forties Marston Morse and Maurice Heins collaborated in a series of papers on the before-mentioned subject. Their aim was two-fold. On the one hand they generalized many theorems from the classical theory of one complex variable to the domain of light interior maps of one Riemann surface to another. Thus they often found topological proofs of topological properties of holomorphic mappings. At the same time they also discovered some remarkable new properties of such mappings, and I would like to describe one of these here. For a more complete survey of their collaboration the reader is referred to Morse's own account [64].

Consider the space $M_{\alpha}$ of all meromorphic maps $f: D \rightarrow S^{2}$ of the disc, $|z|<1$, into the Gauss Sphere subject to the boundary condition

$(\alpha): f$ has a prescribed number of zeros, poles and branch-points of prescribed multiplicity at prescribed points of $D$.

Morse and Heins ask for the components, $\pi_{0}\left(M_{\alpha}\right)$, of $M_{\alpha}$; or, put differently, for the deformation classes of such maps. They then go on to solve this question by constructing a complete set of invariants " $J_{\alpha}$ " characterizing these components. When the multiplicities of all the zeros $a_{0}, \ldots, a_{k}$, and the poles $a_{k+1}, \ldots, a_{n}$, and the branch-points $b_{1}, \ldots, b_{\mu}$ of $\alpha$ are taken to be 1 
the cardinality of the $\left\{J_{\alpha}\right\}$ is $n$, and the invariants $J_{\alpha}(f)$ can be computed for a map $f \in M_{\alpha}$ by the following recipe: One first selects regular $\operatorname{arcs} h_{i}$ joining $a_{0}$ to $a_{i}, i=1, \ldots, n$, which, except for their endpoints, lie in the complement of the $a$ 's and $b$ 's of $\alpha$. It follows that the $f\left(h_{i}\right)$ are regular $\operatorname{arcs}$ on $S^{2}$ which are either closed curves containing the origin, or joining 0 to $\infty$ on $S^{2}$. The recipe for $J_{\alpha}^{i}(f)$-in the first case for instance-is as follows

$$
J_{\alpha}^{i}(f)=\Delta \frac{1}{2 \pi} \arg h_{i}^{\prime}(z)-\Delta \frac{1}{2 \pi} \arg h_{i}(z)-\Delta \frac{1}{2 \pi} \arg C_{i}(z)
$$

where $\Delta$ denotes the algebraic change as $z$ moves on $h_{i}$, and $C_{i}(z)$ is given by

$$
C_{i}(z)=\left[\frac{\left(z-b_{1}\right) \cdots\left(z-b_{n}\right)}{\left(z-a_{1}\right) \cdots\left(z-a_{n}\right)}\right]\left(z-a_{i}\right) .
$$

The $b$ 's here denote the location of the branch-points of $f$, and the virtue of the last term is that it makes $J_{\alpha}^{i}(f)$ independent of the choice of $h_{i}$ joining $a_{0}$ to $a_{i}$.

In the light interior category the same result holds; however there the derivative $h_{i}^{\prime}(z)$ is replaced by a sufficiently short chord. In an earlier paper [61], the authors had already generalized the Whitney theory of regular curves to the domain of locally simple ones, and this classification plays an important role throughout their work.

It seems to me that their result fits into the general framework of the Morse Theory in a way which is not made explicit in their papers, and which would be of contemporary interest. My question is the following one. Recall that any $f \in M_{\alpha}$ is an immersion at all points other than the branch-points, so that its differential $d f$, defines a bundle map

$$
d f: T_{\alpha} D \rightarrow T S^{2}
$$

of the unit tangent bundle of $D$ (with the branch-points deleted) to the unit tangent bundle of $S^{2}$. One may therefore construct a space $\tilde{M}_{\alpha}$ of all continuous bundle-maps from $T_{\alpha} D$ to $T S^{2}$, subject to the boundary conditions $\alpha$, and one then has a natural inclusion

$$
M_{\alpha} \hookrightarrow \tilde{M}_{\alpha}
$$

The Morse-Heins results seem to be equivalent to the assertion that $M_{\alpha}$ and $\tilde{M}_{\alpha}$ have the "same" components-and so the conjecture which comes to mind is that the inclusion (6.2) is a homotopy equivalence. Similar questions seem to be of interest at this time not only to physicists but also to workers in control theory.

7. Integral representations. The very active collaboration of Morse and Transue extended over a period of 10 years or so, starting in 1949. Altogether they produced over 20 papers of considerable complexity, all dealing in one form or another with an extension to bilinear forms of the Riesz representation theorem

$$
F(x)=\int_{0}^{1} x(t) d f
$$

for a continuous linear functional on the space of continuous functions $C$ on $[0,1]$. 
Thus they sought representation for a bilinear function $B(x, y)$ of the form

$$
B(x, y)=\int_{0}^{1} x(s) d_{s} \int_{0}^{1} y(t) d_{t} k(s, t)
$$

where the integrals are taken in the Stieltjes sense.

The original motivation seems to have come from the quadratic form which describes the second variation of an extremal, but $I$ think it is fair to say that after the initial impetus Morse's and Transue's interests became aroused and they explored this territory for its own sake.

The starting point of this work is a (1915) representation theorem for bilinear functions on $C \times C$ due to Fréchet which introduces the notion of Fréchet variation of functions of two variables. In the course of their work Morse and Transue extended this notion to $n$-dimensions and undertook a detailed study of the limit properties of functions of bounded variation [71], [72], [73]. In the process they discovered that in many of the test for convergence of a multiple Fourier series the condition of bounded Vitali variation could be replaced by the weaker condition of bounded Fréchet variation (see [100], [101]), and in the paper [55] they even make a delicate contribution to the convergence theory of Fourier series in one variable. Among other results they show that the Young-Pollard conditions imply the $L_{2}$ conditions of Lebesgue.

8. Pseudo-harmonic-functions. "Among the characteristics of a function $U$ which is harmonic on a Riemann surface $G^{*}$ are the topological interrelations of the level lines of $U$. One merely has to look at the level lines of $R z, R e^{z}$, $R \log z$ to sense both complexity and order. The dual level lines of a conjugate $V$ to $U$ add to this order complexity ..."

This is the beginning sentence of the paper [92], in which Morse and Jenkins solve the difficult problem of showing that on a simply connected Riemann surface every pseudo-harmonic function has a pseudo-conjugate. Thus in particular they show that on such a surface any pseudo-harmonic function can be made harmonic by a change of the conformal structure.

Recall here that pseudo-harmonic means "harmonic after a suitable homeomorphism" so that the topological properties of harmonic functions automatically carry over to pseudo-harmonic ones. In this context $V$ is a pseudo-conjugate to $U$ if there is a homeomorphism of the domain of these functions $\varphi$ such that $(U+i V) \circ \varphi$ is analytic.

The work of Morse and Jenkins extending over the early fifties is devoted to exploring the "order" in the "complexity" mentioned in their "Fundamenta" paper. After essentially setting the simply connected case, where they extended and completed earlier work of Kaplan, Boothby and others, they go on in [94] to discuss these problems on doubly connected surfaces. In particular they there give a very complete analysis of the structure of the level sets of a pseudo-harmonic function.

9. Differential topology. In 1953 M. Morse and E. Baiada wrote a paper [90] which was an assault on the Schoenflies problem by Morse-theoretic means, but it was not until 1959, after B. Mazur's remarkable contribution, that 
Morse became more and more intrigued by the Schoenflies question in all its ramifications.

Most impressive is his 1959 paper [114] where he removes the "unnatural" part of Mazur's hypothesis. Recall that the Schoenflies problem deals with the question of whether a homeomorphism

$$
\varphi: S^{n-1} \rightarrow \mathbf{R}^{n}
$$

of the standard $(n-1)$ sphere into $\mathbf{R}^{n}$ admits an extension to the unit disc $D^{n}$ interior to $S^{n-1}$. The "Horned Sphere" of Alexander shows that in general this is not possible without some tameness assumption on $\varphi$. Essentially untouched for 30 years, Mazur finally decided this question in the affirmative under the following two tameness assumptions:

The first is the natural "shell condition", that $\varphi$ admit a local extension on both sides. Precisely there should exist a homeomorphism

$$
\tilde{\varphi}: S^{n} \times I \rightarrow \mathbf{R}^{n}, \quad I=[0,1],
$$

reducing to $\varphi$ on $S^{n} \times(1 / 2)$.

His second assumption was the less natural one, that $\tilde{\varphi}$ be semilinear near some point $p \in S^{n}$.

To set these conditions in perspective note that if $\varphi$ is smooth, both conditions-with $p$ any point on $S^{n}$-follow immediately. In any case Mazur's inspired construction [M1] led to an easy proof that any $\varphi$ subject to these conditions does admit an extension.

Morse's very fine contribution in [114] was now to remove the second hypothesis, thus achieving the ultimate solution of the Schoenflies problem in the topological setting. By quite different methods the same result was achieved at around the same time by M. Brown [B7].

In his subsequent work on this question-mostly in collaboration with Huebsch, Morse takes up the same question in the smooth and analytic categories, and also considers parameter families of extensions. Thus in [120] they prove the following theorem.

Corresponding to a real analytic mapping

$$
\varphi: S \times \Gamma \rightarrow E
$$

each of whose restrictions to $S \times p, p \in \Gamma$, is an analytic diffeomorphism, there exists an extension

$$
\Lambda: D \times \Gamma \rightarrow E
$$

each of whose restrictions to $D \times p$ is real analytic except possibly for one point.

When $\Gamma$ reduces to a point, this theorem was first established by Royden [R], and to extend it to this parameter form, Huebsch and Morse reproved the Royden result along different lines. Note here that, just as in the $C^{m}$ category, $m>0$, one cannot expect the extension to have smoothness properties at all points of $D$. Indeed the constructions of Milnor produce counterexamples in dimension $\geqslant 6$. On the other hand the iterative constructions of the extension make it fairly clear that the bad behavior of an extension can be concentrated at one point. In [123] Huebsch and Morse introduce the concept of a conical singular point and show how to make extensions whose singular behavior is 
no worse than conical. The article [126] surveys this whole question in considerable detail.

The period 1958-1960 was an exciting one in differential topology. Not only was the Schoenflies problem solved, but Smale [S2] had by that time announced his fundamental results: These included proofs of the Poincaré Conjecture in dimensions $>4$ and of the $h$-cobordism theorem. Both depended on his refined version of the Morse theory, and the resulting handle-body decomposition of manifolds. Morse was of course very pleased with these great successes of the Morse theory. Still, I think he must also have felt a little scooped. In his book with Cairns [CP], he recalls how such handle-bodies were introduced (but not named-or used as spectacularly) by him in an earlier paper. In any case starting in about 1965, and continuing until the late 70's, he undertook to redo this part of differential topology in his own way. Apart from the before mentioned book Morse and Cairns collaborated in many papers on this project. Let me close my account of this phase of Morse's work with a theorem on the removal of critical points which Morse presented in 1965-at a symposium held in his honor-under the title: Bowls of a nondegenerate function on a compact differentiable manifold [139]. This theorem will also serve to introduce us to the ideas of Thom and Smale of the fifties.

The situation envisaged here is that of a nondegenerate function $f$ on a compact manifold $M$. Let the necessarily finite number of critical points of $f$ be denoted by $\operatorname{Cr}(f)$ and consider the complement $\hat{M}=M-\operatorname{Cr}(f)$.

Once a Riemann structure $($,$) is selected on M$, the differential of $f$ uniquely specifies a vector field $X=\hat{d} f$, the gradient of $f$, by the formula

$$
Y \cdot f=(X, Y) \text {. }
$$

This vector field therefore points orthogonally to the level surfaces of $f$ at each point of $M$ and is nonvanishing on $\hat{M}$.

It follows now that the trajectories of $X$ on $\hat{M}$, i.e. the maximal integral manifolds, $l$ of $X$, will be diffeomorphic to the open interval $(0,1)$, and their closures will be curves on $M$ joining two critical points of $f$ at distinct $f$-levels. These are referred to as the lower and upper endpoints of $l$. Now let $p$ be a critical point of $M$. Then Morse calls the point-set consisting of $p$ and all points of trajectories of $X$ with upper endpoint $p$, the "descending Bowl" of $p$, and denotes it by $B^{-}(p)$. Similarly the ascending Bowl, $B^{+}(p)$, is defined by replacing $f$ with $-f$. A first step of the paper under discussion is the theorem that

$A$ Bowl $B^{-}(p)$ is diffeomorphic to $\mathbf{R}^{k}$ with $k$ the index $\lambda(p)$ of $p$.

Thus the Bowls define two decompositions of $M$ into disjoint cells:

$$
\begin{aligned}
& M=\bigcup_{p} B^{-}(p) ; \\
& M=\bigcup_{p} B^{+}(p)
\end{aligned}
$$

with cell dimensions

$$
\operatorname{dim} B^{-}(p)=\lambda(p)
$$




$$
\operatorname{dim} B^{+}(p)=n-\lambda(p) .
$$

REMARKS. The terminology and treatment of this cell-decomposition is somewhat anachronistic. This cell-decomposition already appears in a C-R. Note of Thom's in (1949) [T]. It also plays an essential role in Smale's work of the late 1950s, [S1] and his terminology of stable and unstable manifolds for $B^{ \pm}(p)$ is the generally accepted one. In my own recollection I heard Thom discuss this decomposition in 1951 but its implications were largely lost on me and my contemporaries. Roughly we felt that the flow $X$ became much too complicated "far" from the critical set to be of further use. And locally it, of course, had already been put to use in Theorems A and B. I still remember the surprise therefore when in the late 1950s Smale explained the next step in his program to me. Coming at the Morse Theory from his individual point of view he immediately realized that by perturbing the flow $X$ a little-if necessary-it could be arranged that the two cell decompositions (9.1) and (9.2) be as transversal to each other as possible.

Note in this connection that as both $B^{-}$and $B^{+}$are stable under our flow $X$, the intersection $B^{-} \cap B^{+}$of a descending Bowl from $p$ and an ascending Bowl from $q, p \neq q$, consists of a family of trajectories of $X$. Hence the proper way of defining transversality is to demand that at any point $p \in B^{-} \cap$ $B^{+}$with $f(p)=\alpha$, the intersections of $B^{-}$and $B^{+}$with the level set $M_{\alpha}=\{f$ $=\alpha\}$ in $M$ intersect transversally on $M_{\alpha}$.

These concepts are maybe best understood by studying the two examples of Figure 9.

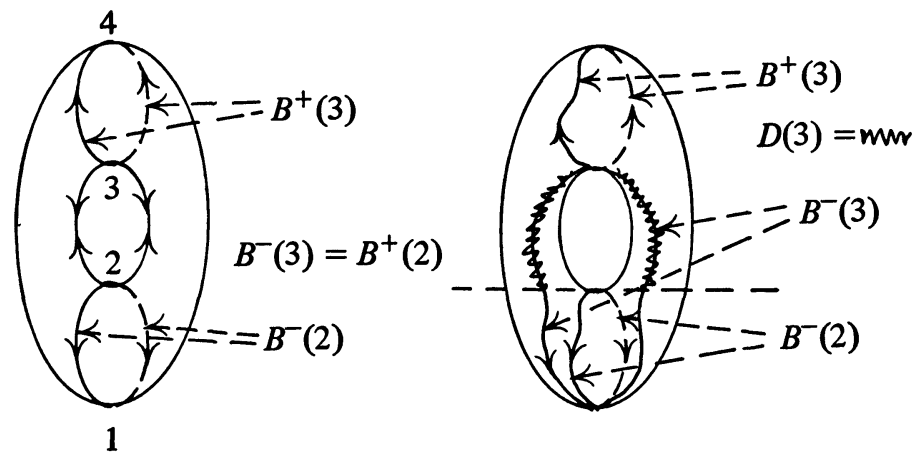

FIgURE 9

On the left we are dealing with the gradient of the $z$-coordinate of a regular torus in $\mathbf{R}^{3}$. The flow it generates is not transversal; for instance $B^{-}(3)$ and $B^{+}(2)$ have no business intersecting at all. The right-hand picture is generic and there they do not.

Note also that $B^{+}(1) \cap B^{-}(3)$ consists of the two trajectories $B^{-}(3)-(3)$.

In his paper [139], Morse constructs a transversal decomposition of $M$ according to a flow $X$ in his own way and then in terms of it formulates and proves a criterion for the elimination of critical points. We still need one additional concept to make this criterion intelligible. Morse calls the "dome" of $a$ "descending Bowl" $B^{-}(p)$, that part of $B^{-}(p)$ where $f$ takes values $>f(c)$ as $c$ ranges over the critical points in the closure of $B^{-}(p)$ other than $p$. In Figure 7 
we have indicated the dome of $B^{-}(3)$ by $D(3)$ on the right-hand figure. The dome of $B^{-}(3)$ is all of $B^{-}(3)$ in the left-hand example.

With this terminology and transversally understood the Bowl-theorem of Morse asserts:

Suppose that $B^{-}(p)$ is a descending $k$ Bowl and $B^{+}(q)$ an ascending $(n-k+1)$ bowl such that $B^{-}(p) \cap B^{+}(q)$ intersect transversally in a single trajectory and such that the closure of $B^{-}(p)$ meets no critical point between the $f$ levels of $p$ and $q$. Also let $N$ be a prescribed open neighborhood of the closure of the dome of $B^{-}(p)$ which contains $p$ and $q$ and such that $\bar{N}$ contains no other critical points of $f$.

Then there exists a nondegenerate function $\hat{f}$ on $M$ without critical points on $\bar{N}$ and such that $\hat{f}=f$ on $M-N$.

For instance in the one-dimensional example of Figure 10 we see that the conditions are satisfied for $B^{-}(1)$ and $B^{+}(2)$ and it should also be clear how to eliminate the critical points 1 and 3. In the generic example of Figure 9 the theorem does not apply-indeed all the pertinent intersections consist of two trajectories. And of course none of these critical points are removable.

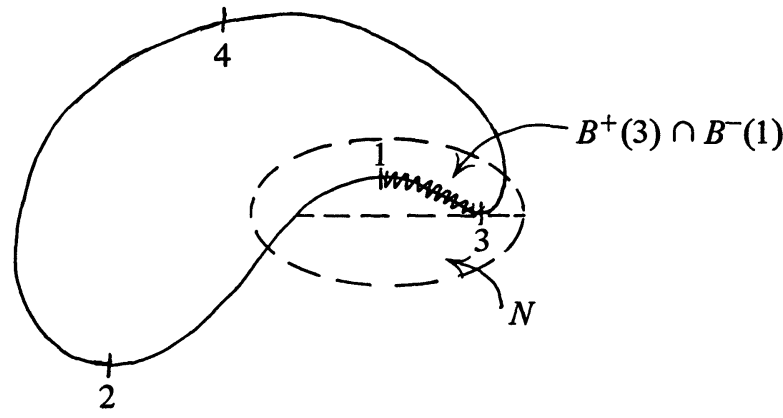

Figure 10

Equipped with this elimination criterion Morse sets to work in this and subsequent work to fashion his version of the whole subject of differential topology. In particular this theorem leads quickly to a result he had established earlier in the difficult paper [113]. The assertion is that on every compact manifold $M$ there exists a nondegenerate function with only one local minimum and one local maximum.

10. Concluding remarks. Alas, there remain many areas of Morse's work which are not accounted for in the previous sections. First of all, there is a wealth of material on the more technical aspects of the calculus of variations. These papers deal with such matters as sufficient conditions for the problem of Meyer and Lagrange under different boundary assumptions, theorems concerning envelopes of extremals, the analytic continuation of closed geodesics, etc. There are also papers on singular quadratic functionals e.g. [34] and related topics. Morse had many collaborators in addition to the ones we have already mentioned: G. Ewing, D. Lander, W. Leighton, G. B. Van Schaack, E. Pitcher-and others. There are also three books (one with S. S. Cairns) and four sets of lecture notes. All in all this magnificent "oeuvre" 
bespeaks Morse's attitude towards his work. Morse was first and foremost a mathematical "craftsman" who did mathematics every day of the year, naturally, and-like Bach-under all conditions; with children on the lap, in the car...

Unfortunately under such a regime there remained little time for nonmathematical writing. Indeed I find only four entries [46], [54], [87], [108] which are not mathematical research or mathematical exposition.

Characteristically all of these deal with the relationship of mathematics to the arts and the practical world. Morse rejoiced in the affinity of mathematics to both, and his views were at once eloquent and optimistic. One meets in these articles a quality not easily gleaned from his purely mathematical writing; a quality which he really showed only to his closer friends and family. Let me conclude therefore with three quotes from these sources.

His Kenyon address [87] starts as follows:

\begin{abstract}
"To talk about art other than in the impersonal sense of history, is to talk about the moments when one has been confronted with beauty. Every essay on art that lights a hidden niche has its source in the life of the writer. You will then perhaps understand why I start with the mood of my childhood. One hundred miles northeast of Derry, New Hampshire, lie the Belgrade Lakes, and out of the last and longest of these lakes flows the Messalonskee. I was born in its valley, 'north of Boston' in the land of Robert Frost."
\end{abstract}

He then continues with a beautiful account of this countryside which he loved so much, and of his early love of music. Then he turns to the art of cabinet making, and in his article on Mathematics, the Arts and Freedom he describes this encounter as follows:

"Turning to another art I recall the shop of an old cabinet-maker on the banks of the Kennebec. It was a place where much could be learned of the relation between mathematics and the arts. Around the room were scattered Sheraton chairs and tables, broken pieces of beauty. Fluted columns, capped by acanthus leaves, made ancient Greece seem near. The cabinetmaker was a provincial Socrates who discoursed with his hands and tools. I wanted to learn more of his art. In a nearby library there was a copy of Sheraton's Cabinet-maker. It started with descriptive geometry and ended with designs of cornices with ruler and compass. Beauty and perfection reigned throughout. Mathematics was the handmaiden of art, faithful if not creative. I did not then realize the difference between mathematics as a servant and as a sister of the arts" (p. 17).

And now listen to how he starts this address:

"Mathematics is an art, and as an art chooses beauty and freedom. It is an aid to technology, but is not a part of technology. It is a handmaiden of the arts, but it is not for this reason an art. Mathematics is an art because its mode of discovery and its inner life are like those of the arts" (p. 16).

One needs first hand knowledge of all these three; the Arts, Mathematics and Freedom, to speak with such authority. 


\section{PUBLICATIONS OF MARSTON MORSE}

\section{PAPERS}

1. Proof of a general theorem on the linear dependence of $P$ analytic functions of a single variable, Bull. Amer. Math. Soc. 23 (1916), 114-117.

2. A one-to-one representation of geodesics on a surface of negative curvature, Amer. J. Math. 43 (1921), 33-51. 84-110.

3. Recurrent geodesics on a surface of negative curvature, Trans. Amer. Math. Soc. 22 (1921),

4. A fundamental class of geodesics on any closed surface of genus p greater than one, Trans. Amer. Math. Soc. 26 (1924), 25-61.

5. Relations between the critical points of a real function of $\boldsymbol{n}$ independent variables, Trans. Amer. Math. Soc. 27 (1925), 345-396.

6. The analysis and analysis situs of regular $n$-spreads in $(n+s)$-space, Proc. Nat. Acad. Sci. 13 (1927), 813-817.

7. The foundations of a theory of the calculus of variations in the large, Trans. Amer. Math. Soc. 30 (1928), 213-274.

8. Singular points of vector fields under general boundary conditions, Amer. J. Math. 51 (1929), 165-178.

9. The critical points of functions and the calculus of variations in the large, Bull. Amer. Math. Soc. 35 (1929), 38-54.

10. The foundations of the calculus of variations in the large in $m$-space (1st paper), Trans. Amer. Math. Soc. 31 (1929), 379-404.

11. Closed extremals, Proc. Nat. Acad. Sci. 15 (1929), 856-959.

12. The problems of Lagrange and Mayer under general end conditions, Proc. Nat. Acad. Sci. 16 (1930), 229-233.

13. A generalization of the Sturm-separation and comparison theorems in n-space, Math. Ann. 103 (1930), 52-69.

14. The critical points of a function of $n$ variables, Proc. Nat. Acad. Sci. 16 (1930), 777-779.

15. The critical points of a function of $n$ variables, Trans. Amer. Math. Soc. 33 (1931), $72-91$.

16. The order of vanishing of the determinant of a conjugate base, Proc. Nat. Acad. Sci. 17 (1931), 319-320.

17. The problems of Lagrange and Mayer with variable endpoints, by $M$. Morse and Sumner Byron Myers, Proc. Amer. Acad. Arts and Sci. 66 (1931), 235-253.

18. Closed extremals (1st paper), Ann. of Math. (2) 32 (1931), 549-566.

19. Sufficient conditions in the problem of Lagrange with fixed endpoints, Ann. of Math. (2) 32 (1931), 567-577.

20. Sufficient conditions in the problem of Lagrange with variable end conditions, Amer. J. Math. 53 (1931), 517-546.

21. The foundations of a theory of the calculus of variations in the large in $m$-space (2nd paper), Trans. Amer. Math. Soc. 32 (1930), 599-631.

22. A characterization of fields in the calculus of variations, by M. Morse and S. B. Littauer, Proc. Nat. Acad. Sci. 18 (1932), 724-730.

23. The calculus of variations in the large, Verhandlungen des Internationalen MathematikerKongresses Zürich, 1932, Vol. 1, pp. 173-188.

24. Does instability imply transitivity?, Proc. Nat. Acad. Sci. 20 (1934), 46-50.

25. On certain invariants of closed extremals, by M. Morse and Everett Pitcher, Proc. Nat. Acad. Sci. 20 (1934), 282-287.

26. The critical point theory under general boundary conditions, by M. Morse and George Booth Van Schaack, Ann. of Math. (2) 35 (1934), 545-571.

27. Sufficient conditions in the problem of Lagrange without assumptions of normalcy, Trans.

Amer. Math. Soc. 37 (1935), 147-160.

28. Instability and transitivity, Jour. de Mathématiques, Paris 14 (1935), 49-71.

29. Abstract critical sets, by M. Morse and George B. Van Schaack, Proc. Nat. Acad. Sci. 21

(1935), 258-263.

30. Generalized concavity theorems, Proc. Nat. Acad. Sci. 21 (1935), 359-362. 
31. Three theorems on the envelope of extremals, Proc. Nat. Acad. Sci. 21 (1935), 619-621; Bull. Amer. Math. Soc. 42 (1936), 136-144.

32. Functional topology and abstract variational theory, Proc. Nat. Acad. Sci. 22 (1936), 313-319.

33. Critical point theory under general boundary conditions, by M. Morse and George B. Van Schaack, Duke Math. J. 2 (1936), 220-242.

34. Singular quadratic functionals, by M. Morse and Walter Leighton, Trans. Amer. Math. Soc. 40 (1936), 252-286.

35. A special parametrization of curves, Bull. Amer. Math. Soc. 42 (1936), 915-922.

36. Functional topology and abstract variational theory, Ann. of Math. (2) 38 (1937), 386-449.

37. The index theorem in the calculus of variations, Duke Math. J. 4 (1938), 231-246.

38. Functional topology and abstract variational theory, Proc. Nat. Acad. Sci. 24 (1938), 326-330.

39. Symbolic dynamics, by M. Morse and G. A. Hedlund, Amer. J. Math. 60 (1938), 815-866.

40. On the existence of minimal surfaces of general critical types, by M. Morse and C. B.

Tompkins, Proc. Nat. Acad. Sci. 25 (1939), 153-158; Ann. of Math. (2) 40 (1939),443-472.

41. Sur le calcul des variations, Ann. Inst. H. Poincaré 9 (1939), 1-11.

42. La dynamique symbolique, Bull. Soc. Math. France 67 (1939), 1-7.

43. Symbolic dynamics. II. Sturmian sequences, by M. Morse and G. A. Hedlund, Amer. J. Math. 61 (1940), 1-42.

44. Rank and span in functional topology, Ann. of Math. (2) 41 (1940), 419-454.

45. The first variation in minimal surface theory, Duke Math. J. 6 (1940), 263-289.

46. Twentieth century mathematics, Amer. Scholar 9 (1940), 499-504.

47. Unstable minimal surfaces of higher topological types, by M. Morse and C. B. Tompkins, Proc. Nat. Acad. Sci. 26 (1940), 713-716.

48. A mathematical theory of equilibrium with applications to minimal surface theory, Science 93 (1941), 69-71.

49. Minimal surfaces not of minimum type by a new mode of approximation, by $M$. Morse and

C. B. Tompkins, Ann. of Math. (2) 42 (1941), 62-72.

50. Corrections to our paper on the existence of minimal surfaces of general critical types, by $\mathbf{M}$.

Morse and C. B. Tompkins, Ann. of Math. (2) 42 (1941), 331.

51. Mathematics in the defense program, by M. Morse and W. L. Hart, The Math. Teacher, May 1941, pp. 195-202.

52. Unstable minimal surfaces of higher topological structure, by M. Morse and C. B. Tompkins, Duke Math. J. 8 (1941), 350-375.

53. The continuity of the area of harmonic surfaces as a function of the boundary representations,

by M. Morse and C. B. Tompkins, Amer. J. Math. 63 (1941), 825-838.

54. Report on the War Preparedness Committee of the AMS and MAA at the Chicago meeting,

Bull. Amer. Math. Soc. 47 (1941), 829-831.

55. What is analysis in the large?, Amer. Math. Monthly 49 (1942), 358-364.

56. Manifolds without conjugate points, by M. Morse and G. A. Hedlund, Trans. Amer. Math. Soc. 51 (1942), 362-386.

57. Mathematics and the maximum scientific effort in total war, Scientific Monthly 56 (1943), 1-6.

58. Variational theory in the large including the non-regular case, by $\mathbf{M}$. Morse and George Ewing-First paper, Ann. of Math. (2) 44 (1943), 339-353; Second paper, Ann. of Math. (2) 44 (1943), 354-374.

59. Functional topology, Bull. Amer. Math. Soc. 49 (1943), 144-149.

60. Unending chess, symbolic dynamics and a problem in semigroups, by M. Morse and G. A. Hedlund, Duke Math. J. 11 (1944), 1-7.

61. Topological methods in the theory of functions of a single complex variable: I. Deformation types of locally simple plane curves; II. Boundary values and integral characteristics of interior transformations and pseudoharmonic functions, by M. Morse and M. Heins, Ann. of Math. (2) 46 (1945), 600-624; 625-666; III. Causal isomorphisms in the theory of pseudoharmonic functions, Ann. of Math. (2) 47 (1946), 233-273.

62. The topology of pseudoharmonic functions, Duke Math. J. 13 (1946), 21-42.

63. George David Birkhoff and his mathematical work, Bull. Amer. Math. Soc. 52 (1946), 357-391. 
64. Topological methods in the theory of functions of a complex variable, by M. Morse and $\mathbf{M}$. Heins, Bull. Amer. Math. Soc. 53 (1947), 1-15.

65. Deformation classes of meromorphic functions and their extensions to interior transformations, by M. Morse and M. Heins, Acta Math. 79 (1947), 51-103.

66. Functions on a metric space and a setting for isoperimetric problems, Studies and Essays, presented to $R$. Courant on his 60th birthday, January 8, 1948, pp. 253-263. Interscience Publishers Inc., New York, 1948.

67. A positive, lower semicontinuous nondegenerate function on a metric space, Fund. Math. 35 (1948), 47-78.

68. L-S-homotopy classes of locally simple curves, Ann. Soc. Polonaise Math. 21 (1948), 236-256.

69. Equilibria in nature, stable and unstable, Proc. Amer. Philos. Soc. 93 (1949), 222-225.

70. The Fréchet variation and the convergence of multiple Fourier series, by $\mathbf{M}$. Morse and W. Transue, Proc. Nat. Acad. Sci. 35 (1949), 395-399.

71. Integral representations of bilinear functionals, by $\mathbf{M}$. Morse and W. Transue, Proc. Nat. Acad. Sci. 35 (1949), 136-143.

72. Functionals of bounded Fréchet variation, by M. Morse and W. Transue, Canad. J. Math. 1 (1949), 153-165.

73. Functionals $F$ bilinear over the product $A \times B$ of two p-normed vector spaces: 1 . The representation of F, by M. Morse and W. Transue, Ann. of Math. (2) 50 (1949), 777-815; II. Admissible spaces A, Ann. of Math. (2) 51 (1950), 576-614.

74. Les progrès de l'analyse variationnelle globale et son programme, Rendiconti di Matematica e delle sue applicazioni, Serie V, 70 (3) (4) (1948), 1-11.

75. Topological methods in the theory of functions of a complex variable, Ann. Matematica 28 (1949), 21-25.

76. A characterization of the bilinear sum associated with the classical second variation, by $\mathbf{M}$. Morse and W. Transue, Ann. Matematica 29 (1949), 25-68.

77. L-S homotopy classes on the topological image of the projective plane, Bull. Amer. Math. Soc. 55 (1949), 981-1003.

78. The Fréchet variation and a generalization for multiple Fourier series of the Jordan test, by M. Morse and W. Transue, Rivista di Matematica della Università di Parma 1 (1950), 1-16.

79. The Fréchet variation sector limits, and left decompositions, by M. Morse with W. Transue, Canad. J. Math. (3) 2 (1950), 344-374.

80. A calculus of Fréchet variations, by M. Morse with W. Transue, J. Indian Math. Soc. 14 (2) (3) (1950), 65-117.

81. The Fréchet variation and Pringsheim convergence of double Fourier series, Contributions to Fourier Analysis, by M. Morse and W. Transue, Ann. Math. Studies, no. 25, Princeton Univ. Press, Princeton, N. J., 1950, pp. 46-103.

82. Norms of distribution functions associated with bilinear functionals, by M. Morse and W. Transue, ibidem, pp. 104-144.

83. Bilinear functionals over CXC, by M. Morse and W. Transue, Acta Sci. Math. 12 (1950), $41-48$.

84. Recent advances in variational theory in the large, Proc. Internat. Cong. Math. 2 (1950), 143-156.

85. A new implication of the Young-Pollard convergence criteria for a Fourier series, by $\mathbf{M}$.

Morse and W. Transue, Duke Math. J. 18 (1951), 563-571.

86. Trends in analysis, J. Franklin Inst. 251 (1951), 33-43.

87. Mathematics and the arts, Yale Review 40 (1951), 604-612. 75th birthday of Robert Frost. Essay given at Kenyon College.

88. Homology relations on regular orientable manifolds, Proc. Nat. Acad. Sci. 38 (1952), 247-258.

89. Contour equivalent pseudoharmonic functions and pseudoconjugates, by $\mathbf{M}$. Morse with J. Jenkins, Amer. J. Math. 74 (1952), 23-51.

90. Homotopy and homology related to the Schoenflies problem, by M. Morse with E. Baiada, Ann. of Math. (2) 58 (1953), 142-165.

91. Topological methods on Riemann surfaces. Pseudoharmonic functions, by M. Morse with J. Jenkins, Ann. of Math. Studies, no. 30, Princeton Univ. Press, Princeton, N. J., 1953, pp. 111-139. 
92. The existence of pseudoconjugates on Riemann surfaces, by M. Morse with J. Jenkins, Fund. Math. 39 (1953), 269-287.

93. The generalized Fréchet variation and Riesz-Young-Hausdorff type theorems, by M. Morse with W. Transue, Rend. Circ. Math. Palermo, Serie II, Tome II (1953), 35 pp.

94. Conjugate nets, conformal structure, and interior transformations on open Riemann surfaces, by M. Morse with J. Jenkins, Proc. Nat. Acad. Sci. 39 (1953), 1261-1268.

95. Conjugate nets on an open Riemann surface, by M. Morse with J. Jenkins, Proc. Univ. Michigan Conf., June 1953.

96. Curve families $F^{*}$ locally the level curves of a pseudoharmonic function, by M. Morse with J. Jenkins, Acta Math. 91 (1954), 42 pp.

97. (With W. Transue) Semi-normed vector spaces with duals of integral type, J. Analyse Math. 4 (1954-1955), 149-186.

98. Bimeasures and their integral extensions, Ann. Mat. Pura Appl. 39 (1955), 345-356.

99. (With W. Transue) $C$-bimeasures $\Lambda$ and their superior integrals $\Lambda^{*}$, Rend. Circ. Math. Palermo 4 (1955), 270-300.

100. (With W. Transue) The representation of a $C$-bimeasure on a general rectangle, Proc. Nat. Acad. Sci. 42 (1956), 89-95.

101. La construction topologique d'un réseau isotherme sur une surface ouverte, J. Math. Pures Appl. 35 (1956), 67-75.

102. (With W. Transue) $C$-bimeasures $\Lambda$ and their integral extensions, Ann. of Math. (2) 64 (1956), 480-504.

103. (With W. Transue) Products of a $C$-measure and a locally integrable mapping, Canad. J. Math. 9 (1957), 475-486.

104. Differentiable mappings in the Schoenflies problem, Proc. Nat. Acad. Sci. 44 (1958), 1068-1072.

105. (With W. Transue) Vector subspaces $A$ of $C^{E}$ with duals of integral type, J. Math. 37 (1958), 44-363.

106. (With W. Transue) The existence of vector function spaces with duals of integral type, Colloq. Math. 6 (1958), 95-117.

107. (With W. Transue) The local characterization of vector function spaces with duals of integral type, J. Analyse Math. 6 (1958), 225-260.

108. Mathematics and the arts, Bull. of the Atomic Scientists 15 (1959), 55-59 (Yale Rev. 1951).

109. Mathematics, the arts and freedom, Thought (Fordham Univ. Quarterly) 34 (1959), 16-24.

110. Differentiable mappings in the Schoenflies theorem, Comp. Math. 14 (1959), 83-151.

111. Topologically nondegenerate functions on a compact n-manifold $M$, J. Analyse Math. 7 (1959), 189-208.

112. Fields of geodesics issuing from a point, Proc. Nat. Acad. Sci. 46 (1960), 105-111.

113. The existence of polar nondegenerate functions on differentiable manifolds, Ann of Math. (2) 71 (1960), 352-383.

114. A reduction of the Schoenflies extension problem, Bull. Amer. Math. Soc. 66 (1960), 113-115.

115. The existence of nondegenerate functions on a compact differentiable m-manifold $M, A n n$. Mat. Pura Appl. 49 (1960), 117-128.

116. Recurrent geodesics on a surface of negative curvature, Trans. Amer. Math. Soc. 22 (1921), 84-110. (Multilithed with Historical Note, 1960.)

117. (With W. Huebsch) An explicit solution of the Schoenflies extension problem, J. Math. Soc. Japan 12 (1960), 271-289.

118. (With W. Huebsch) A singularity in the Schoenflies extension, Proc. Nat. Acad. Sci. 46 (1960), 1100-1102.

119. On elevating manifold differentiability, J. Indian Math. Soc. 24 (1960), 379-400.

120. (With W. Huebsch) The dependence of the Schenflies extension on an accessory parameter, J. Analyse Math. 8 (1960-1961), 209-271.

121. (With W. Huebsch) Abstracts: $A$ characterization of an analytic $n$-ball, and A. Schoenflies extension of a real analytic diffeomorphism of $S$ into $W$. (Multilithed April 17, 1961.)

122. Boundary values of partial derivatives of Poisson integral, Ann. Acad. Brasil. Ci. 33 (1961), 131-139.

123. (With W. Huebsch) Conical singular points of diffeomorphisms, Bull. Amer. Math. Soc. 67 (1961), 490-493. 
124. (With W. Huebsch) Schoenflies extensions of analytic families of diffeomorphisms, Math. Ann. 144 (1961), 162-174.

125. (With W. Huebsch) The Schoenflies extension in the analytic case, Ann. Mat. Pura Appl. 54 (1961), 359-378.

126. Schoenflies problems, Fund. Math. 50 (1962), 319-332.

127. (With W. Huebsch) Schoenflies extensions without interior differential singularities, Ann. of Math. (2) 76 (1962), 18-54.

128. Topological, differential, and analytic formulations of Schoenflies problems, Rend. Circ. Mat. Palermo 21 (1962), 286-295. (Lecture delivered in Rome, April 1962.)

129. (With W. Huebsch) Analytic diffeomorphisms approximating $C^{m}$-diffeomorphisms, Rend. Circ. Mat. Palermo 11 (1962), 1-22.

130. (With W. Huebsch) Diffeomorphisms of manifolds, Rend. Circ. Mat. Palermo 11 (1962), 1-28.

131. Schoenflies extensions and differentiable isotopies, J. Mat. Pures Appl. 42 (1963), 29-41.

132. An arbitrarily small analytic mapping into $R_{+}$of a proper, regular, analytic r-manifold in $E_{m}$, Rendi. Sci. Ist. Lombardo, Milano (A) 97 (1963), 650-660.

133. Harmonic extensions, Monatsh. Math. 67 (1963), 317-325.

134. (With W. Huebsch) The dependence of the Shoenflies extension on an accessory parameter (the topological case), Proc. Nat. Acad. Sci. 50 (1963), 1036-1037.

135. (With W. Huebsch) The bowl theorem and a model nondegenerate function, Proc. Nat. Acad. Sci. 51 (1964), 49-51.

136. (With W. Huebsch) Conditioned differentiable isotopies, Differential Analysis Colloq., Bombay, India, 1964, pp. 1-25.

137. The elimination of critical points of a nondegenerate function on a differentiable manifold, $\mathrm{J}$. Analyse Math. 13 (1964), 257-316.

138. Bowls, f-fibre bundles and the alteration of critical values, Ann. Acad. Brasil. Ci. 36 (1964), 245-259.

139. Bowls of a nondegenerate function on a compact differentiable manifold, Differential and Combinatorial Topology, Princeton Univ. Press, Princeton, N. J., 1965, pp. 81-103. (Symposium in honor of M. Morse.)

140. Quadratic forms $\Theta$ and $\Theta$-fibre bundles, Ann. of Math. (2) 81 (1965), 303-340.

141. (With W. Huebsch), A model nondegenerate function, Revue Roumaine Math. Pures Appl. 10 (1965), 691-722.

142. The reduction of a function near a nondegenerate critical point, Proc. Nat. Acad. Sci. 54 (1965), 1759-1764.

143. Projective methods (in photogrammetry), Photogrammetric Engineering, Sept. 1966, pp. 849-855.

144. (With J. Cantwell) Diffeomorphism including automorphisms of $\pi_{1}\left(T_{p}\right)$, Topology 4 (1966), 323-341.

145. Nondegenerate functions on abstract differentiable manifolds $M$, J. Analyse Math. 19 (1967), 231-272.

146. Nondegenerate real-valued differentiable functions, Proc. Nat. Acad. Sci. 57 (1967), 32-38.

147. Focal sets of regular manifolds $M_{n-1}$ in $E_{n}$, J. Differential Geometry 1 (1967), 1-19.

148. Bowls, $f$-fiber bundles and the alteration of critical values, Proc. Nat. Acad. Sci. 60 (1968), 1156-1159.

149. (With S. S. Cairns) Singular homology over $\mathbf{Z}$ on topological manifolds, J. Differential Geometry 3 (1969), 257-288.

150. (With S. S. Cairns) A setting for a theorem of Bott, Proc. Nat. Acad. Sci. 65 (1970), 8-9.

151. Mathematics in our culture, The Spirit and the Uses of the Mathematical Sciences by T. L. Saaty and F. J. Weyl, McGraw-Hill, New York, 1969, pp. 105-120.

152. Equilibrium points of harmonic potentials, J. Analyse Math. 23 (1970), 281-296.

153. (With S. S. Cairns) Elementary quotients of abelian groups, and singular homology on manifolds, Nagoya Math. J. 39 (1970), 167-198.

154. Subordinate quadratic forms and their complementary forms, Proc. Nat. Acad. Sci. 68 (1971), 579.

155. Model families of quadratic forms, Proc. Nat. Acad. Sci. 68 (1971), 914-915.

156. (With S. S. Cairns) Orientation of differentiable manifolds, J. Differential Geometry 6 (1971), 1-31. (Dedicated to S. S. Chern and D. C. Spencer.) 
157. Subordinate quadratic forms and their complementary forms, Rev. Roumaine Math. Pures Appl. 16 (1971), 559-569.

158. (With S. S. Cairns) Singular homology on an untriangulated manifold, J. Differential Geometry 7 (1972), 1-17.

159. Axial presentations of regular arcs on $M_{n}$, Proc. Nat. Acad. Sci. 69 (1972), 3504-3505.

160. Singular quadratic functionals, Math. Ann. 201 (1973), 315-340.

161. F-deformations and F-tractions, Proc. Nat. Acad. Sci. 70 (1973), 1634-1635.

162. Fréchet curve classes, J. Math. Pures Appl. 53 (1974), 291-298.

163. Singleton critical values, Bull. Inst. Math. Acad. Sinica 2 (1974), 317-333.

164. (With D. Landis) Geodesic joins and Fréchet curve classes, Rend. Mat. 8 (1975), 161-185.

165. Connectivities $R_{i}$ of Fréchet spaces in variational topology, Proc. Nat. Acad. Sci. 72 (1975), 2069-2070.

166. Topologically nondegenerate functions, Fund. Math. 88 (1975), 17-52.

167. (With D. Landis) Tractions in critical point theory, Rocky Mountain J. Math. 5 (1975), 379-399.

168. Fréchet numbers in global variational analysis, Houston J. Math. 2 (1976), 387-403.

169. (With S. S. Cairns) Frechet numbers and geodesics on surfaces, Bull. Inst. Math. Acad. Sinica 4 (1976), 7-34.

170. Conjugate points on a limiting extremal, Proc. Nat. Acad. Sci. 73 (1976), 1800-1801.

171. Extremal limits of nondegenerate extremals, Rend. Mat. 9 (1976), 621-632.

172. Tubular presentations $\pi$ of subsets of manifolds, Proc. Nat. Acad. Sci. 74 (1977), 2209-2210.

173. Nondegenerate point pairs in global variational analysis, J. Differential Geometry 11 (1976), 617-632.

174. Uses of the Frechet numbers $R_{i}\left(M_{n}\right)$ of a smooth manifold, Houston J. Math. 3 (1977), 503-513.

175. Lacunary type number sequence in global analyses, J. Math. Pures Appl. 57 (1978), 87-98.

176. The Frechet numbers of the differentiable product of two compact converted smooth manifolds, Edited and completed by S. S. Cairns, Univ. of Illinois, for Marston Morse memorial issue of Bull. of Institute of Math. Acad. Sinica, Vol. VI, No. 2, October 1978.

\section{Books}

1. Calculus of variations in the large, Amer. Math. Soc. Colloq. Publ., no. 18, Amer. Math. Soc., Providence, R. I., 1934. (Address: P. O. Box 6248, Providence, R. I., 02904.)

2. Functional topology and abstract variational theory, Mémorial des Sciences Mathématiques 92, Gauthier-Villars, Paris, 1939.

3. Topological methods in the theory of functions of a complex variable, Princeton Univ. Press, Princeton, N. J., 1947. (Paperback available from Kraus Reprint Co., 16 E. 46th St., New York, N. Y. 10017.)

4. Symbolic dynamics, Lectures of 1938 with New Preface, 1966, 87 pp. (Notes by R. Oldenburger.) University Microfilms, 300 N. Zeeb Road, Ann Arbor, Mich. 48106.

5. (With S. S. Cairns) Critical point theory in global analysis and differential topology, Academic Press, New York, 1969.

6. Variational analysis: Critical extremals and Sturmian extensions, Wiley, New York, 1973.

7. Global variational analysis: Weierstrass integrals on a Riemannian manifold, Mathematical Notes, Princeton Univ. Press, Princeton, N. J., 1976.

\section{BIBLIOGRAPHY}

[A] A. Andreotti and T. Frankel, The Lefschetz theorem on hyperplane sections, Ann. of Math. (2) 69 (1959), 713-717.

[B1] G. D. Birkhoff, Dynamical systems with two degrees of freedom, Trans. Amer. Math. Soc. $18(1917), 240$.

[B2] , Quelques théorèmes sur le mouvement des systèmes dynamiques, Bull. Soc. Math. France 40 (1912), 305-323. 
[B3] G. D. Birkhoff and M. R. Hestenes, Generalized minimax principle in the calculus of variations, Duke Math. J. 1 (1935).

[B4] R. Bott, Nondegenerate critical manifolds, Ann. of Math. (2) 60 (1954), 248-261.

[B5] __ The stable homotopy of the classical groups, Ann. of Math. (2) 70 (1959), 179-203.

[B6] R. Bott and H. Samelson, Applications of the theory of Morse to symmetric spaces, Amer. J. Math. 80 (1958), 964-1029.

[B7] Morton Brown, A proof of the generalized Schoenflies theorem, Bull. Amer. Math. Soc. 66 (1960), 74-76.

[D1] R. Deheuvels, Topologie d'une fonctionelle, Ann. of Math. (2) 61 (1955), 13-72.

[H1] J. Hadamard, Les surfaces à courbure opposées et leur lignes géodesiques, J. Math. Pures Appl. (5) 4 (1898).

[H2] G. Hedlund, On the metrical transitivity of geodesics on closed surfaces of constant negative curvature, Ann. of Math. (2) 35 (1934), 787-808.

[K] W. Klingenberg, Lectures on closed geodesics, Grundlehren der Mathematischen Wissenschaften, Springer-Verlag.

[M1] B. Mazur, On embeddings of spheres, Bull. Amer. Math. Soc. 65 (1959), 59-65.

[M2] J. Milnor, Morse theory, Ann. of Math. Studies no. 51, Princeton Univ. Press, Princeton, N. J., 1963.

[N1] P. Novikov, One periodic groups, Dokl. Akad. Nauk SSSR 127 (1959), 749-752; English transl., Amer. Math. Soc. Transl. (2) 45 (1965), 19-22.

[P1] R. Palais, Morse theory on Hilbert manifolds, Topology 2 (1963), 299-340.

[R] H. L. Royden, The analytic approximation of differentiable mappings, Math. Ann. 139 (1960), 171-179.

[S1] Seifert-Threlfall, Variationsrechnung im Grossen, Teubner, Leipzig, 1938.

[S2] S. Smale, Generalized Poincaré conjecture in higher dimensions, Bull. Amer. Math. Soc. 66 (1960), 373-375.

[S3] __ A survey of some recent developments in differential topology, Bull. Amer. Math. Soc. 69 (1963), 131-145.

[S4] __ Differentiable dynamical systems, Bull. Amer. Math. Soc. 73 (1967), 747-817.

[S5] G. Series, Symbolic dynamics for geodesic flows, Univ. Warwick, 1979.

[T1] R. Thom, Sur une partition en cellules associée á une fonction sur une variété, C. R. Acad. Sci. Paris 228 (1949), 973-975.

Department of Mathematics, Harvard University, Cambridge, Massachusetts 02138 